\title{
BPS solutions of six-dimensional $(1,0)$ supergravity coupled to tensor multiplets
}

\section{Huibert het Lam and Stefan Vandoren}

Institute for Theoretical Physics and Centre for Extreme Matter and Emergent Phenomena, Utrecht University,

3508 TD Utrecht, The Netherlands

E-mail: H.hetLam@uu.nl, S.J.G.Vandoren@uu.nl

ABSTRACT: We derive a general local form for supersymmetric solutions of six-dimensional $(1,0)$ supergravity coupled to an arbitrary number of tensor multiplets. We consider some special cases in which the resulting equations can be solved explicitly. In particular we derive black string solutions and calculate their entropy. Upon reducing to five dimensions they yield spinning black hole solutions. We also discuss BPS pp-waves and black string solutions with traveling waves. Lastly, as an application, we study the attractor mechanism in this theory.

Keywords: Supersymmetric Effective Theories, Supergravity Models, Black Holes

ARXiv EPrint: 1804.04681 


\section{Contents}

1 Introduction 1

2 Setting 4

$2.1 N=1$ restrictions 5

$\begin{array}{lll}2.2 & \text { Equations of motion } & 7\end{array}$

$\begin{array}{lll}3 & \text { Supersymmetric solutions } & 7\end{array}$

3.1 Introduction of coordinates 8

$\begin{array}{ll}3.2 & \text { Supersymmetry and self-duality }\end{array}$

$\begin{array}{lll}3.3 & \text { Bianchi identity } & 10\end{array}$

$\begin{array}{lll}3.4 & \text { Einstein equation } & 10\end{array}$

$\begin{array}{lll}3.5 & \text { Summary } & 11\end{array}$

4 Classes of solutions $\quad 11$

4.1 Non-twisting solutions 11

$4.2 u$-independent solutions $\quad 14$

$\begin{array}{lll}4.3 & \text { Reduction to five dimensions } & 15\end{array}$

$\begin{array}{ll}4.4 \text { Gibbons-Hawking base space } & 17\end{array}$

4.5 Black strings and other objects with a horizon 21

4.6 pp-waves 24

5 Attractor mechanism $\quad \mathbf{2 6}$

$\begin{array}{lll}5.1 & \text { Near horizon analysis } & 26\end{array}$

$\begin{array}{lll}5.2 \text { Flow equation } & 26\end{array}$

5.3 One-centered Gibbons-Hawking 28

$\begin{array}{llr}6 & \text { Outlook } & 29\end{array}$

$\begin{array}{ll}\text { A Integrability conditions } & 30\end{array}$

$\begin{array}{ll}\text { B Spin connection } & 31\end{array}$

$\begin{array}{lll}\text { C } R_{--} \text {component of the Ricci tensor } & 31\end{array}$

D Single string solution $\quad 32$

D.1 Asymptotics 33

E Reduction of the flow equation to five dimensions 33 


\section{Introduction}

The phase space of (quantum) gravity solutions in dimensions larger than four is intricate and has a rich structure, see e.g. [1, 2] for some reviews on black holes and horizons in various dimensions. Whereas in asymptotically flat four spacetime dimensions, horizon topologies are unique to be $S^{2}$, in five dimensions one can have black holes and black rings [3], with horizons $S^{3}$ and $S^{1} \times S^{2}$ respectively. Also quotient topologies exist, such as Lens space horizons [4-6]. These objects can be made BPS in supergravity and are embeddable in string theory where one can provide a microscopic description. In six dimensions, near horizon geometries have been classified in $(1,0)$ supergravity coupled to tensor multiplets and hypermultiplets (and no vector multiplets) [7, 8], and there are more possibilities. The focus of this paper, the case where the hypermultiplets are frozen, only yields near horizon geometries locally given by either $\mathbb{R}^{1,1} \times T^{4}, \mathbb{R}^{1,1} \times K 3$ or $A d S_{3} \times S^{3}$. Some of the objects with a horizon easily follow from uplifts from $5 \mathrm{~d}$ to $6 \mathrm{~d}$. The most well studied case is of course the 6d BPS black string with horizon $S^{1} \times S^{3}$ and near horizon geometry $A d S_{3} \times S^{3}$, arising from the uplift of a $5 \mathrm{~d}$ spherical black hole. The microscopic description in string theory was first given in $[9,10]$. One can also uplift a 5d black ring and we will see that it also has horizon $S^{1} \times S^{3}$ and near horizon geometry $A d S_{3} \times S^{3}$. Besides black objects with horizons, there are also smooth horizonless BPS (microstate-) geometries (see [11-16] for an incomplete list of references), and an interesting class of $6 \mathrm{~d}$ BPS pp-wave solutions that we will study in this paper. Many other solutions can be found by superposing waves on black strings, and one can play with various kinds of asymptotics.

So far general BPS solutions of $(1,0)$ supergravity have mainly been studied in the minimal case [7] and in the case with the gravity multiplet coupled to one or two tensor multiplets (gauged and ungauged), see e.g. [11-20]. In this paper we generalize this to $n_{T}$ tensor multiplets, for any $n_{T}$. One of the new ingredients is the scalars in the tensor multiplets which can have nontrivial profiles and that are subject to a $6 \mathrm{~d}$ attractor mechanism, as we will see.

Six-dimensional $(1,0)$ supergravity coupled to matter multiplets arises from the compactification of F-theory on elliptically fibered Calabi-Yau threefolds or from truncations of type IIB on $T^{4}$ or $K_{3}$. Of particular interest are BPS black string solutions of this theory, as they yield five-dimensional black holes upon further compactification on a circle. The F-theory microscopics have been studied in [21-23]. The near horizon geometry leads to new two-dimensional $(0,4)$ CFTs that have been recently investigated in $[22,24,25]$. Compactifying F-theory on an elliptically fibered non-singular Calabi-Yau threefold results in a gravity multiplet, $n_{T}=h^{1,1}\left(B_{2}\right)-1$ tensor multiplets and $n_{H}=h^{2,1}\left(C Y_{3}\right)+1$ hypermultiplets, where $h^{1,1}\left(B_{2}\right)$ and $h^{2,1}\left(C Y_{3}\right)$ are hodge numbers of the base and threefold respectively [26-28]. For generic elliptic fibrations, one has $n_{V}=h^{1,1}\left(C Y_{3}\right)-h^{1,1}\left(B_{2}\right)-1$ vector multiplets and an anomaly cancelation condition. Here we truncate all vectors and set the corresponding charges to zero. One approach to study F-theory is via its connection with M-theory: one gets the effective $6 \mathrm{~d}(1,0)$ theory by reducing M-theory on the Calabi-Yau threefold to five dimensions and then lifting it up to six dimensions [29]. To 
do this, one has to take into account one-loop contributions coming from the reduction on the circle [30]. So in a way six-dimensional solutions are proper F-theory solutions and it might be interesting to see what we can learn from studying the connection between them and their five-dimensional counterparts.

More technically, our analysis starts with deriving a general local form for supersymmetric solutions where we use methods that have been applied before to four-dimensional theories [31-36], five-dimensional theories [37-40] and minimal six-dimensional supergravity [7]. The strategy is always the same. Starting from a Killing spinor $\epsilon$ one constructs bosonic objects quadratic in $\epsilon$, the so-called bilinears, such as the vector $X^{\mu}=\bar{\epsilon} \gamma^{\mu} \epsilon$. These bilinears have certain properties since $\epsilon$ is a Killing spinor. The vector $X^{\mu}$ for instance turns out to be a Killing vector in all cases. Using these bilinears, the local form of the solutions can be identified and this can be used to simplify the equations of motion. It turns out that the resulting equations in minimal $N=2, D=4$ supergravity can actually be solved completely [32]. In five-dimensional minimal supergravity the solutions fall into two classes [37]. In the first class, the vector $X$ is null and the solutions are plane-fronted waves expressed in harmonic functions on $\mathbb{R}^{3}$. In the second class, the vector $X$ is timelike and the equations of motion can not be solved completely, but the equations are simplified significantly such that one only has to make an ansatz for the remaining variables. Solutions of supergravity coupled to an arbitrary number of vector multiplets have similar properties as in the minimal case [39]. In six-dimensional minimal supergravity the Killing vector $X$ is always null [7] and as one can expect, based on the five-dimensional analysis, the equations of motion can not be solved completely in the most general case.

The bosonic field content in the six-dimensional theory consists of the metric, a twoform with anti-self-dual field strength in the gravity multiplet, and $n_{T}$ two-forms with self-dual field strength and $n_{T}$ scalars in the tensor multiplets. The constraints that supersymmetry puts on the field content have already been derived in [41], so like in [7] we will introduce coordinates and reduce the equations of motion using these local expressions. Using coordinates $\left(u, v, x^{m}\right), m=1, \ldots, 4$ we find that solutions are not dependent on $v$ and can be expressed in terms of a $u$-dependent base manifold $\mathcal{B}$. In general the base space $\mathcal{B}$ exhibits a non-integrable hyper-Kähler structure. Using the form of the solutions that one gets from requiring one Killing spinor, all the equations can be expressed in terms of bosonic quantities on $\mathcal{B}$. These equations are not easy to solve in full generality, but it is still easier to find solutions by substituting an ansatz in these equations than to start with an ansatz for the complete field content.

The resulting equations will be studied in two cases where the base space becomes hyper-Kähler. One of those cases arises when the solution is $u$-independent. In that case we also take $\mathcal{B}$ to be Gibbons-Hawking [42] and it turns out that the solution is completely determined in terms of $6+2 n_{T}$ harmonic functions on $\mathbb{R}^{3}$. This is one of the main new results of our analysis. When one takes these solutions to be multi-centered GibbonsHawking, requiring the absence of Dirac string-like singularities gives restrictions on the relative positions of the centers. Just as in the minimal case [43] there is a symplectic group that sends solutions to solutions preserving regularity, but here the $\operatorname{Sp}(6)$ gets enlarged to $\operatorname{Sp}\left(6+2 n_{T}\right)$. We also construct the macroscopic black string solutions in F-theory 
backgrounds $\mathbb{R} \times S^{1} \times \mathbb{R}^{4} \times C Y_{3}$ with a D3-brane wrapped on $S^{1} \times C$, where $C$ is a curve in the base of the Calabi-Yau threefold.

We finish by studying the attractor mechanism $[44,45]$ in this theory. This has partly been done in $[46,47]$ but only the near horizon analysis. Here we derive a flow equation on the tensor branch for $u$-independent solutions. We look at simplifying assumptions needed to understand the attractor values as the optimization of the central charge, and we apply this to the one-centered Gibbons-Hawking class of solutions that include BPS black strings. Some of the latter solutions have also been treated in [48].

This paper is organized as follows. In section 2 we describe the field content of $(1,0)$ supergravity with a gravity multiplet and tensor multiplets, we list the conditions the existence of a Killing spinor puts on the field content, and we introduce the equations of motion that are not implied by integrability conditions. In section 3 we introduce coordinates and reduce the equations of motion to equations in terms of bosonic quantities on $\mathcal{B}$. Section 4 then solves the resulting equations under certain assumptions. Here we also discuss how the theory reduces to five dimensions and look at black strings and other objects with a horizon. We finish this section by looking at examples of pp-waves. Section 5 describes the attractor mechanism for this theory; here we also derive a flow equation for $u$-independent solutions. In section 6 we then summarize and give some suggestions for future work.

Notation. Since we use a lot of different notation in this paper, we give a short overview:

- $M, N=1, \ldots, n_{T}$ where $n_{T}$ is the number of tensor multiplets,

- $\alpha, \beta, \ldots=1, \ldots, n_{T}+1$ denote field content in six dimensions,

- $\mu, \nu, \ldots=0, \ldots, 5$ denote coordinates or the vielbein of the six-dimensional metric,

- $i, j, k, l=1, \ldots, 4$ first denote part of the vielbein of the six-dimensional space and from section 3 onwards the vielbein of the base manifold $\mathcal{B}$,

- $m, n, \ldots=1, \ldots, 4$ denote the coordinates of the base manifold $\mathcal{B}$,

- $a, b, c, d=1, \ldots, 3$ denote either the three two-forms in the almost hyper-Kähler structure, or part of the vielbein of a Gibbons-Hawking metric,

- $I, J, \ldots=0, \ldots, n_{T}+1$ denote field content in five dimensions,

- objects with a hat $\hat{\cdot}$ live in six dimensions,

- objects with a tilde ? live in the four-dimensional base space,

- $\star_{6}$ is the hodge star in six dimensions,

- $\star_{4}$ is the hodge star in the four-dimensional base space.

Note added. During the submission process of this paper, we learned of similar and independent work [49] that has some overlap with ours. 


\section{Setting}

We consider six-dimensional $(1,0)$ supergravity coupled to $n_{T}$ tensor multiplets [50-52]. Vector and hypermultiplets can be added to ensure an F-theory embedding, but we will set them to zero in the solutions we consider in this paper. The idea is to study the BPS structure of the tensor branch. The bosonic content of the gravity multiplet consists of a graviton and a two-form with anti-self-dual field strength. Every tensor multiplet contains a two-form with self-dual field strength and a scalar. We will denote the sixdimensional two-forms by $\hat{B}^{\alpha}$, where $\alpha=1, \ldots, n_{T}+1$. The scalars of the tensor multiplets parametrize the coset space $\mathrm{SO}\left(1, n_{T}\right) / \mathrm{SO}\left(n_{T}\right)$. A convenient way to describe them is by an $\mathrm{SO}\left(1, n_{T}\right)$ matrix

$$
S=\left(\begin{array}{c}
j_{\alpha} \\
x_{\alpha}^{M}
\end{array}\right), M=1, \ldots, n_{T},
$$

whose matrix elements satisfy the constraints

$$
\begin{aligned}
j_{\alpha} j_{\beta}-\sum_{M} x_{\alpha}^{M} x_{\beta}^{M} & =\Omega_{\alpha \beta}, \\
j_{\alpha} j^{\alpha} & =1, \\
x_{\alpha}^{M} j^{\alpha} & =0,
\end{aligned}
$$

where $\Omega_{\alpha \beta}=\operatorname{diag}(1,-1, \ldots,-1)$ is used to lower and raise $\alpha$ indices. The field strengths corresponding to the two-forms are given by $\hat{G}^{\alpha}=d \hat{B}^{\alpha}$ and their relation to the anti-selfdual tensor $H$ of the gravity multiplet and the self-dual tensors $H^{M}$ of the tensor multiplets is given by

$$
\hat{G}^{\alpha}=j^{\alpha} H-\Omega^{\alpha \beta} \sum_{M} x_{\beta}^{M} H^{M} .
$$

In terms of the three-forms $\hat{G}^{\alpha}$ the self-duality condition can be written as

$$
g_{\alpha \beta}{ }_{6} \hat{G}^{\beta}=-\Omega_{\alpha \beta} \hat{G}^{\beta},
$$

where

$$
g_{\alpha \beta}=2 j_{\alpha} j_{\beta}-\Omega_{\alpha \beta}
$$

is a positive definite metric.

The self-duality condition on the three-forms makes it hard to construct a covariant action functional from which all equations of motion follow. These actions do exist [53, 54], but one has to introduce auxiliary fields. Another approach is a pseudo-action from which equations of motion follow that then still have to be supplemented by the self-duality condition. The bosonic part of the pseudo-action that is relevant for this paper is given by $[52]$

$$
S=\int_{\mathcal{M}_{6}} \frac{1}{2} R \star_{6} 1-\frac{1}{4} g_{\alpha \beta} \hat{G}^{\alpha} \wedge \star_{6} \hat{G}^{\beta}+\frac{1}{2} \Omega_{\alpha \beta} d j^{\alpha} \wedge \star_{6} d j^{\beta}
$$


The equations of motion are then equal to

$$
\begin{aligned}
R_{\mu \nu} & =\frac{1}{4} g_{\alpha \beta} \hat{G}_{\mu}^{\alpha \rho \lambda} \hat{G}_{\nu \rho \lambda}^{\beta}-\frac{1}{24} \hat{g}_{\mu \nu} g_{\alpha \beta} \hat{G}_{\rho \lambda \sigma}^{\alpha} \hat{G}^{\beta \rho \lambda \sigma}-\Omega_{\alpha \beta} \partial_{\mu} j^{\alpha} \partial_{\nu} j^{\beta}, \\
x_{\alpha}^{M} d\left(\star_{6} d j^{\alpha}\right) & =-x_{\alpha}^{M} j_{\beta} \hat{G}^{\alpha} \wedge \star_{6} \hat{G}^{\beta}, \\
d\left(g_{\alpha \beta} \star_{6} \hat{G}^{\beta}\right) & =0, \\
g_{\alpha \beta} \star_{6} \hat{G}^{\beta} & =-\Omega_{\alpha \beta} \hat{G}^{\beta}, \\
j_{\alpha} j^{\alpha} & =1 .
\end{aligned}
$$

Using the self-duality condition, this set of equations is equivalent to

$$
\begin{aligned}
R_{\mu \nu} & =\frac{1}{4} g_{\alpha \beta} \hat{G}_{\mu}^{\alpha \rho \lambda} \hat{G}_{\nu \rho \lambda}^{\beta}-\Omega_{\alpha \beta} \partial_{\mu} j^{\alpha} \partial_{\nu} j^{\beta}, \\
x_{\alpha}^{M} d\left(\star_{6} d j^{\alpha}\right) & =x_{\alpha}^{M} j_{\beta} \hat{G}^{\alpha} \wedge \hat{G}^{\beta}, \\
d \hat{G}^{\alpha} & =0 \\
g_{\alpha \beta} \star_{6} \hat{G}^{\beta} & =-\Omega_{\alpha \beta} \hat{G}^{\beta} \\
j_{\alpha} j^{\alpha} & =1 .
\end{aligned}
$$

Note that the equation of motion for the three-forms becomes the Bianchi identity.

\section{1 $\quad N=1$ restrictions}

The BPS equations for six-dimensional $(1,0)$ supergravity with a gravity multiplet and tensor multiplets are [41]

$$
\begin{aligned}
\left(\nabla_{\mu}-\frac{1}{8} H_{\mu \nu \rho} \gamma^{\nu \rho}\right) \epsilon & =0, \\
\left(\frac{i}{2} T_{\mu}^{M} \gamma^{\mu}-\frac{i}{24} H_{\mu \nu \rho}^{M} \gamma^{\mu \nu \rho}\right) \epsilon & =0,
\end{aligned}
$$

where

$$
T_{\mu}^{M} \equiv x_{\alpha}^{M} \partial_{\mu} j^{\alpha}
$$

We will consider geometries with $N=1$ supersymmetry. In [41] the restrictions that the Killing spinor puts on the geometry have been worked out which we will discuss in this section.

On our geometry we introduce a vielbein $\hat{e}^{0}, \ldots, \hat{e}^{5}$, the null-forms ${ }^{1}$

$$
\begin{aligned}
e^{-} & =\frac{1}{\sqrt{2}}\left(-\hat{e}^{0}+\hat{e}^{5}\right), \\
e^{+} & =\frac{1}{\sqrt{2}}\left(\hat{e}^{0}+\hat{e}^{5}\right)
\end{aligned}
$$

and choose the orientation $\epsilon^{-+1234}=1$. Using the null-vielbein $e^{-}, e^{+}, \hat{e}^{i}$, where $i=$ $1, \ldots, 4$, we can write the metric as

$$
d s_{6}^{2}=2 e^{-} e^{+}+\delta_{i j} \hat{e}^{i} \hat{e}^{j} .
$$

\footnotetext{
${ }^{1}$ We relabeled the vielbein of [41] to make notation easier later on.
} 
We choose the orientation of the directions perpendicular to the light-cone direction $\epsilon^{i j k l}=$ $\epsilon^{-+i j k l}$ (note that [41] uses the opposite orientation).

The Killing spinor can then be used to construct the bilinears of the geometry, which turn out to be given by $e^{-}$and

$$
e^{-} \wedge I^{1}, \quad e^{-} \wedge I^{2}, \quad e^{-} \wedge I^{3},
$$

where the two-forms $I^{a}$ for $a \in\{1,2,3\}$ take the form

$$
\begin{aligned}
& I^{1}=-\left(\hat{e}^{1} \wedge \hat{e}^{3}+\hat{e}^{2} \wedge \hat{e}^{4}\right), \\
& I^{2}=-\left(\hat{e}^{1} \wedge \hat{e}^{2}-\hat{e}^{3} \wedge \hat{e}^{4}\right), \\
& I^{3}=-\left(\hat{e}^{1} \wedge \hat{e}^{4}-\hat{e}^{2} \wedge \hat{e}^{3}\right) .
\end{aligned}
$$

These two-forms are anti-self-dual on the directions perpendicular to the light-cone direction and satisfy the algebra of the imaginary unit quaternions on a four-manifold with metric $\delta_{i j} \hat{e}^{i} \hat{e}^{j}$ :

$$
\left(I^{a}\right)_{k}^{i}\left(I^{b}\right)^{k}=-\delta^{a b} \delta_{j}^{i}+\epsilon^{a b}{ }_{c}\left(I^{c}\right)^{i}{ }_{j} .
$$

Note that the vierbein chosen in (2.14) is special and that the forms $I^{a}$ might look different when another vierbein is used.

The conditions that the gravitino Killing spinor equation imposes on the spacetime geometry can be rewritten as

$$
\begin{aligned}
\nabla_{\mu} e_{\nu}^{-} & =\frac{1}{2} H_{\mu \nu}^{\lambda} e_{\lambda}^{-} \\
\nabla_{\mu}\left(e^{-} \wedge I^{a}\right)_{\nu \lambda \rho} & =\frac{1}{2} H_{\mu \nu}^{\sigma}\left(e^{-} \wedge I^{a}\right)_{\sigma \lambda \rho}+\frac{1}{2} H_{\mu \lambda}^{\sigma}\left(e^{-} \wedge I^{a}\right)_{\nu \sigma \rho}+\frac{1}{2} H_{\mu \rho}^{\sigma}\left(e^{-} \wedge I^{a}\right)_{\nu \lambda \sigma}
\end{aligned}
$$

Condition (2.16) implies that the vector $X$ dual to $e^{-}$is a Killing vector. Furthermore, condition (2.16), the $\mu=-$ component of condition (2.17) and the anti-self-duality of $H$ imply that

$$
H=e^{+} \wedge d e^{-}-\frac{1}{16}\left(I_{k l}^{a} \nabla_{-} I^{b k l}\right) \epsilon_{a b}^{c} I_{c i j} e^{-} \wedge \hat{e}^{i} \wedge \hat{e}^{j}+\frac{1}{6}\left(d e^{-}\right)_{-l} \epsilon_{i j k}^{l} \hat{e}^{i} \wedge \hat{e}^{j} \wedge \hat{e}^{k} .
$$

The $\mu=+$ component of condition (2.17) implies that

$$
\nabla_{+} I^{a}=0
$$

and the $\mu=i$ components give further restrictions on the covariant derivatives of $I^{a}$. The tensorini Killing spinor equation implies that the scalars of the tensor multiplets are invariant under the isometry $X[41]$. Combining this equation with the self-duality condition yields that the three-forms of the tensor multiplets take the following form [41]:

$$
H^{M}=\frac{1}{2} H_{-i j}^{M} e^{-} \wedge \hat{e}^{i} \wedge \hat{e}^{j}+T_{i}^{M} e^{-} \wedge e^{+} \wedge \hat{e}^{i}+\frac{1}{6} T_{l}^{M} \epsilon_{i j k}^{l} \hat{e}^{i} \wedge \hat{e}^{j} \wedge \hat{e}^{k}
$$


where $\frac{1}{2} H_{-j k}^{M} e^{-} \wedge \hat{e}^{j} \wedge \hat{e}^{k}$ are self-dual tensors on the directions transverse to the light cone part.

Notice that

$$
\sum_{M} x_{\beta}^{M} T_{i}^{M}=-\Omega_{\alpha \beta} \partial_{i} j^{\alpha}
$$

From (2.3), (2.18) and (2.20) we then find that

$$
\begin{aligned}
\hat{G}^{\alpha}= & j^{\alpha} e^{+} \wedge d e^{-}+e^{-} \wedge e^{+} \wedge d j^{\alpha}+\frac{1}{6}\left[j^{\alpha}\left(d e^{-}\right)_{-l}+\left(d j^{\alpha}\right)_{l}\right] \epsilon_{i j k}^{l} \hat{e}^{i} \wedge \hat{e}^{j} \wedge \hat{e}^{k} \\
& -\left[\frac{1}{8} j^{\alpha}\left(I_{k l}^{a} \nabla_{-} I^{b k l}\right) \epsilon_{a b}^{c}\right] e^{-} \wedge I_{c}+e^{-} \wedge H_{\mathrm{SD}}^{\alpha}
\end{aligned}
$$

where

$$
H_{\mathrm{SD}}^{\alpha} \equiv-\frac{1}{2} \Omega^{\alpha \beta} \sum_{M} x_{\beta}^{M} H_{-i j}^{M} \hat{e}^{i} \wedge \hat{e}^{j}
$$

is a self-dual two-form on the directions transverse to the light cone part. Notice that $j_{\alpha} H_{\mathrm{SD}}^{\alpha}=0$. Using the Bianchi identity we calculate

$$
\mathcal{L}_{X} \hat{G}^{\alpha}=i_{X} d \hat{G}^{\alpha}+d i_{X} \hat{G}^{\alpha}=d\left(j^{\alpha} d e^{-}+d j^{\alpha} \wedge e^{-}\right)=0 .
$$

Condition (2.17) combined with the anti-self-duality of $H$ and $I^{a}$ imply that $d\left(e^{-} \wedge I^{a}\right)=0$ such that also $\mathcal{L}_{X}\left(e^{-} \wedge I^{a}\right)=0$. Hence $X$ generates a symmetry of the full solution.

Summary. Using the vielbein $e^{-}, e^{+}, \hat{e}^{i}$ the field content takes the form (2.12) and (2.22). Furthermore, we have the two-forms (2.14) that satisfy the condition (2.19) and the $\mu=i$ components of (2.17). The null-vector $X$ dual to $e^{-}$is a symmetry of the full solution.

\subsection{Equations of motion}

From the integrability conditions it follows that if the BPS equations (2.9) and the Bianchi identity are satisfied, the scalar equations of motion and all but the -- component of the Einstein equation are obeyed (see appendix A). Hence the set of equations of motion (2.8) reduces to

$$
\begin{aligned}
R_{--} & =\frac{1}{4} g_{\alpha \beta} \hat{G}_{-}^{\alpha \rho \lambda} \hat{G}_{-\rho \lambda}^{\beta}-\Omega_{\alpha \beta} \partial_{-} j^{\alpha} \partial_{-} j^{\beta}, \\
d \hat{G}^{\alpha} & =0, \\
g_{\alpha \beta} \star_{6} \hat{G}^{\beta} & =-\Omega_{\alpha \beta} \hat{G}^{\beta}, \\
j_{\alpha} j^{\alpha} & =1 .
\end{aligned}
$$

Note that in the solution of the BPS equations (2.22), the self-duality condition has already been taken care of.

\section{Supersymmetric solutions}

In this section we first use the existence of a null Killing vector to introduce coordinates on the geometry. After this, we rewrite the Bianchi identity and the Einstein equation using these coordinates. 


\subsection{Introduction of coordinates}

The existence of a null Killing vector $X$ can be used to introduce coordinates on our spacetime. We implement this here following similar steps as in [7]. First a hypersurface $\mathcal{S}$ has to be picked that is nowhere tangent to $X$. One then has to pick a vector $Y$ that satisfies

$$
\hat{g}(Y, X)=1, \quad \hat{g}(Y, Y)=0,
$$

on $\mathcal{S}$. This vector $Y$ needs to be propagated off $\mathcal{S}$ by solving $\mathcal{L}_{X} Y=0$. The properties (3.1) still hold since $X$ is Killing. The vectors $X$ and $Y$ commute, hence they must be tangent to a four-parameter family of two-dimensional surfaces $\Sigma_{2}\left(x^{m}\right)$, where $m=1, \ldots, 4$. The vector $X$ is a null Killing vector, so should be tangent to affinely parametrized null geodesics. Define the coordinate $v$ to be this affine parameter along the geodesics and choose another coordinate $u$ such that $u, v$ are coordinates on the surfaces $\Sigma_{2}$. We can then write

$$
\begin{aligned}
& X=\partial_{v}, \\
& Y=H\left(\partial_{u}-\frac{1}{2} \mathcal{F} \partial_{v}\right),
\end{aligned}
$$

for functions ${ }^{2} H$ and $\mathcal{F}$ independent of $v$. We will assume that (locally) $H>0$ since we can send $u \rightarrow-u$ if necessary. Using the properties of $X$ and $Y$ it can be shown [7] that the metric takes the form

$$
d s_{6}^{2}=2 H^{-1}(d u+\beta)\left(d v+\omega+\frac{1}{2} \mathcal{F}(d u+\beta)\right)+H d s_{4}^{2},
$$

where $d s_{4}^{2}=h_{m n} d x^{m} d x^{n}$ is the metric of a base space $\mathcal{B}$ and $\omega, \beta$ are one-forms on $\mathcal{B}$ [7]. The functions $H$ and $\mathcal{F}$, the one-forms $\omega$ and $\beta$ and the metric $h_{m n}$ only depend on $u$ and $x^{m}$ (since $X$ is a Killing vector). The one-forms $e^{-}, e^{+}$in (2.12) take the form

$$
\begin{aligned}
e^{-} & =H^{-1}(d u+\beta), \\
e^{+} & =d v+\omega+\frac{1}{2} \mathcal{F} H e^{-} .
\end{aligned}
$$

Notice also that the relation between $\hat{e}^{i}$ and a vierbein $\tilde{e}^{i}$ of $\mathcal{B}$ is given by $\hat{e}^{i}=H^{1 / 2} \tilde{e}^{i}$ which implies $\partial_{\hat{i}}=H^{-1 / 2} \partial_{i}$. From now on, the labels $i, j, k, l$ will refer to the vielbein on the base space.

Let us define anti-self-dual forms on $\mathcal{B}$ by

$$
J^{a}=H^{-1} I^{a} .
$$

These satisfy the algebra

$$
\left(J^{a}\right)_{p}^{m}\left(J^{b}\right)_{n}^{p}=\epsilon_{c}^{a b}\left(J^{c}\right)_{n}^{m}-\delta^{a b} \delta_{n}^{m},
$$

where the indices $m, n, \ldots$ have been raised with $h^{m n}$. Hence, these two-forms yield an almost hyper-Kähler structure on $\mathcal{B}$.

\footnotetext{
${ }^{2}$ This notation might be a bit confusing since we already used $H$ for the three-form in the gravity multiplet, but to more easily compare with other literature, we will keep it.
} 
Following [7] we introduce some more notation. Suppose $\Phi$ is a $p$-form with all its legs on $\mathcal{B}$, but with coefficients depending on $u$ (denote this by $\Phi \in \Lambda^{p}(\mathcal{B})(u)$ ):

$$
\Phi=\frac{1}{p !} \Phi_{m_{1} \ldots m_{p}}(u, x) d x^{m_{1}} \wedge \ldots \wedge d x^{m_{p}}
$$

We then introduce the restricted exterior derivative $\tilde{d}$ by

$$
\tilde{d} \Phi \equiv \frac{1}{(p+1) !}(p+1) \frac{\partial}{\partial x^{[n}} \Phi_{\left.m_{1} \ldots m_{p}\right]} d x^{n} \wedge d x^{m_{1}} \wedge \ldots \wedge d x^{m_{p}} .
$$

We also define the operator $\mathcal{D}$ acting on such $p$-forms as

$$
\mathcal{D} \Phi=\tilde{d} \Phi-\beta \wedge \dot{\Phi},
$$

where $\dot{\Phi}$ is the Lie derivative of $\Phi$ with respect to $\partial_{u}$. Note that

$$
d \Phi=\mathcal{D} \Phi+H e^{-} \wedge \dot{\Phi} .
$$

Also $\mathcal{D}$ obeys the same product rule on wedge products as the exterior derivative and

$$
\mathcal{D}^{2} \Phi=-\mathcal{D} \beta \wedge \dot{\Phi}
$$

Using these operators we can derive that

$$
\begin{aligned}
& d e^{-}=H^{-1} \mathcal{D} \beta+e^{-} \wedge\left(H^{-1} \mathcal{D} H+\dot{\beta}\right), \\
& d e^{+}=\mathcal{D} \omega+\frac{1}{2} \mathcal{F} \mathcal{D} \beta+H e^{-} \wedge\left(\dot{\omega}+\frac{1}{2} \mathcal{F} \dot{\beta}-\frac{1}{2} \mathcal{D} \mathcal{F}\right) .
\end{aligned}
$$

From these expressions it is straightforward to calculate the spin connection components. They can be found in appendix B.

\subsection{Supersymmetry and self-duality}

We now derive what the conditions of section 2.1 become in terms of the coordinates we introduced. The three-forms (2.22) become

$$
\begin{aligned}
\hat{G}^{\alpha}= & e^{+} \wedge e^{-} \wedge\left[j^{\alpha}\left(H^{-1} \mathcal{D} H+\dot{\beta}\right)-\mathcal{D} j^{\alpha}\right]+j^{\alpha} H^{-1} e^{+} \wedge \mathcal{D} \beta \\
& +e^{-} \wedge\left[j^{\alpha} H \psi-j^{\alpha}(\mathcal{D} \omega)^{-}+H_{\mathrm{SD}}^{\alpha}\right]+\star_{4} \mathcal{D}\left(j^{\alpha} H\right)+H j^{\alpha} \star_{4} \dot{\beta},
\end{aligned}
$$

where $(\mathcal{D} \omega)^{-} \equiv \frac{1}{2}\left(\mathcal{D} \omega-\star_{4} \mathcal{D} \omega\right), \star_{4}$ is the Hodge dual on $\mathcal{B}$ and

$$
\psi \equiv-\frac{1}{8} H \epsilon_{a b c} J_{k l}^{a} \partial_{u}\left(J^{b k l}\right) J^{c} .
$$

The self-duality condition (2.27) implies that

$$
\begin{aligned}
\mathcal{D} \beta & =\star_{4} \mathcal{D} \beta, \\
H_{\mathrm{SD}}^{\alpha} & =\star_{4} H_{\mathrm{SD}}^{\alpha} .
\end{aligned}
$$


Using this one can show that (2.19) is satisfied. The remaining constraints are the $\mu=$ $i$ components of (2.17) and they constrain the covariant derivatives of $J^{a}$ on $\mathcal{B}$. Since $d\left(e^{-} \wedge I^{a}\right)=0$ we find that

$$
\tilde{d} J^{a}=\mathcal{L}_{\partial_{u}}\left(\beta \wedge J^{a}\right)
$$

Together with the fact that the $J^{a}$ satisfy the algebra (3.6) this implies the $\mu=i$ components of (2.17). From (3.17) we conclude that the almost hyper-Kähler structure of $\mathcal{B}$ is not integrable in general.

\subsection{Bianchi identity}

We can now substitute expression (3.13) for the three-forms in the Bianchi identity $d \hat{G}^{\alpha}=$ 0 . Note that this equation is also the equation of motion of the three-forms because of the self-duality condition. Using (3.12), the Bianchi identity reduces to

$$
\tilde{d}\left(j^{\alpha} \psi+\mathcal{G}^{+\alpha}\right)=\mathcal{L}_{\partial_{u}}\left[\beta \wedge\left(j^{\alpha} \psi+\mathcal{G}^{+\alpha}\right)+\star_{4} \mathcal{D}\left(j^{\alpha} H\right)+H j^{\alpha} \star_{4} \dot{\beta}\right],
$$

and

$$
\mathcal{D} \star_{4}\left[\mathcal{D}\left(j^{\alpha} H\right)+H j^{\alpha} \dot{\beta}\right]+\mathcal{D} \beta \wedge \mathcal{G}^{+\alpha}=0,
$$

where we defined the self-dual two-forms

$$
\mathcal{G}^{+\alpha}=H^{-1}\left[j^{\alpha}(\mathcal{D} \omega)^{+}+\frac{1}{2} j^{\alpha} \mathcal{F} \mathcal{D} \beta+H_{\mathrm{SD}}^{\alpha}\right],
$$

with $(\mathcal{D} \omega)^{+} \equiv \frac{1}{2}\left(\mathcal{D} \omega+\star_{4} \mathcal{D} \omega\right)$.

\subsection{Einstein equation}

We now consider (2.25). In appendix $\mathrm{C}$ we use the spin connection components to calculate that

$$
\begin{aligned}
R_{--}= & \star_{4} \mathcal{D} \star_{4}\left(\dot{\omega}+\frac{1}{2} \mathcal{F} \dot{\beta}-\frac{1}{2} \mathcal{D} \mathcal{F}\right)-2\left(\dot{\omega}+\frac{1}{2} \mathcal{F} \dot{\beta}-\frac{1}{2} \mathcal{D} \mathcal{F}\right)^{m} \partial_{u}\left(\beta_{m}\right) \\
& +\frac{1}{2} H^{-2}\left(\mathcal{D} \omega+\frac{1}{2} \mathcal{F} \mathcal{D} \beta\right)^{2}-\frac{1}{2} H h^{m n} \partial_{u}^{2}\left(H h_{m n}\right)-\frac{1}{4} \partial_{u}\left(H h^{m n}\right) \partial_{u}\left(H h_{m n}\right),
\end{aligned}
$$

where for $\Phi_{1}, \Phi_{2} \in \Lambda^{2}(\mathcal{B})(u), \Phi_{1} \cdot \Phi_{2}=\frac{1}{2} \Phi_{1 m n} \Phi_{2}^{m n}$. From (3.13) we find that

$$
\frac{1}{4} g_{\alpha \beta} \hat{G}_{-}^{\alpha \rho \lambda} \hat{G}_{-\rho \lambda}^{\beta}=\frac{1}{2}\left[\psi-H^{-1}(\mathcal{D} \omega)^{-}\right]^{2}+\frac{1}{2} H^{-2} g_{\alpha \beta} H_{\mathrm{SD}}^{\alpha} \cdot H_{\mathrm{SD}}^{\beta} .
$$

The scalars do not depend on $v$ so

$$
\partial_{-} j^{\alpha}=H \partial_{u} j^{\alpha}
$$

and the Einstein equation becomes

$$
\begin{aligned}
\star_{4} \mathcal{D} \star_{4}\left(\dot{\omega}+\frac{1}{2} \mathcal{F} \dot{\beta}-\frac{1}{2} \mathcal{D} \mathcal{F}\right)= & 2\left(\dot{\omega}+\frac{1}{2} \mathcal{F} \dot{\beta}-\frac{1}{2} \mathcal{D} \mathcal{F}\right)^{m} \partial_{u}\left(\beta_{m}\right)+\frac{1}{2} H h^{m n} \partial_{u}^{2}\left(H h_{m n}\right) \\
& +\frac{1}{4} \partial_{u}\left(H h^{m n}\right) \partial_{u}\left(H h_{m n}\right)-\frac{1}{2} H^{-2}\left(\mathcal{D} \omega+\frac{1}{2} \mathcal{F} \mathcal{D} \beta\right)^{2} \\
& +\frac{1}{2}\left[\psi-H^{-1}(\mathcal{D} \omega)^{-}\right]^{2}+\frac{1}{2} H^{-2} g_{\alpha \beta} H_{\mathrm{SD}}^{\alpha} \cdot H_{\mathrm{SD}}^{\beta} \\
& -\Omega_{\alpha \beta} H^{2} \partial_{u} j^{\alpha} \partial_{u} j^{\beta}
\end{aligned}
$$




\subsection{Summary}

We derived the general local form of all supersymmetric solutions of six-dimensional $(1,0)$ supergravity with a gravity multiplet and $n_{T}$ tensor multiplets. The metric is given by (3.3) and the three-forms by (3.13). The equations of motion can be reduced to a set of equations on the base manifold $\mathcal{B}$. The one-form $\beta$ and two-forms $H_{\mathrm{SD}}^{\alpha}$ must obey the self-duality conditions (3.15) and (3.16). The Bianchi identity and Einstein equation reduce to (3.18) and (3.19), and (3.24) respectively. The base manifold must admit an almost hyper-Kähler structure with almost complex structures obeying (3.17).

\section{Classes of solutions}

In this section we will, following [7], consider two cases in which the equations derived in the previous section reduce considerably. We first focus on so-called non-twisting solutions, which are solutions in which $\beta=0$. After that we look at $u$-independent solutions and consider their reduction to five dimensions. When the base-space of a $u$-independent solution is chosen to be Gibbons-Hawking and the symmetry of this metric is extended to be a symmetry of the whole solution, we show that it can be expressed in $6+2 n_{T}$ harmonic functions on $\mathbb{R}^{3}$. We then briefly investigate the multi-centered Gibbons-Hawking subclass of these solutions and look at objects as the black string that have a horizon. We finish this section with some examples of pp-wave solutions.

\subsection{Non-twisting solutions}

Non-twisting solutions have $\beta=0$ which highly simplifies the equations. The metric (3.3) and three-forms (3.13) reduce to

$$
d s_{6}^{2}=2 H^{-1} d u\left(d v+\omega+\frac{1}{2} \mathcal{F} d u\right)+H d s_{4}^{2},
$$

and

$$
\hat{G}^{\alpha}=-e^{+} \wedge d u \wedge \tilde{d}\left(H^{-1} j^{\alpha}\right)+H^{-1} d u \wedge\left[j^{\alpha} H \psi-j^{\alpha}(\tilde{d} \omega)^{-}+H_{\mathrm{SD}}^{\alpha}\right]+\star_{4} \tilde{d}\left(j^{\alpha} H\right) .
$$

The base space $\mathcal{B}$ with metric $d s_{4}^{2}$ has to be hyper-Kähler since from (3.17) it follows that

$$
\tilde{d} J^{a}=0 .
$$

The Bianchi identity, (3.18) and (3.19), reduce to

$$
\tilde{d}\left(j^{\alpha} \psi+\mathcal{G}^{+\alpha}\right)=\mathcal{L}_{\partial_{u} \star_{4}} \tilde{d}\left(j^{\alpha} H\right),
$$

and

$$
\tilde{\nabla}^{2}\left(j^{\alpha} H\right)=0
$$

where

$$
\mathcal{G}^{+\alpha}=H^{-1}\left[j^{\alpha}(\tilde{d} \omega)^{+}+H_{\mathrm{SD}}^{\alpha}\right]
$$


Hence the functions $j^{\alpha} H$ are harmonic. The Einstein equation (3.24) reduces to

$$
\begin{aligned}
\tilde{\nabla}^{i}(\dot{\omega})_{i}-\frac{1}{2} \tilde{\nabla}^{2} \mathcal{F}= & \frac{1}{2} H h^{m n} \partial_{u}^{2}\left(H h_{m n}\right)+\frac{1}{4} \partial_{u}\left(H h^{m n}\right) \partial_{u}\left(H h_{m n}\right)-\frac{1}{2} H^{-2}(\tilde{d} \omega)^{2} \\
& +\frac{1}{2}\left[\psi-H^{-1}(\tilde{d} \omega)^{-}\right]^{2}+\frac{1}{2} H^{-2} g_{\alpha \beta} H_{\mathrm{SD}}^{\alpha} \cdot H_{\mathrm{SD}}^{\beta}-\Omega_{\alpha \beta} H^{2} \partial_{u} j^{\alpha} \partial_{u} j^{\beta} .
\end{aligned}
$$

In principle one should be able to solve these equations successively. First pick a hyperKähler base space $\mathcal{B}$ and pick harmonic functions on this space for $j^{\alpha} H$. The function $H$ then follows by using the identity $j_{\alpha} j^{\alpha}=1$. The two-form $\mathcal{G}^{+\alpha}$ can be determined by using its self-duality and (4.4). Then $\omega$ can be determined by contracting (4.6) with $j_{\alpha}$ which then also gives an expression for $H_{\mathrm{SD}}^{\alpha}$. Lastly, $\mathcal{F}$ can be determined from (4.7).

Solutions that are dependent on $u$ (but not necessarily non-twisting) have been studied in the case of minimal supergravity or in the case with an extra tensor multiplet $[13,19,55-$ 57]. To show that one can still do this with an arbitrary number of tensor multiplets, we construct an explicit example of a $u$-dependent solution.

Flat base space. We will extend the non-twisting solution of [7] with flat base space to a solution with tensor multiplets. As metric on the base space we take

$$
d s_{4}^{2}=d r^{2}+\frac{1}{4} r^{2}\left[\left(\sigma^{1}\right)^{2}+\left(\sigma^{2}\right)^{2}+\left(\sigma^{3}\right)^{2}\right],
$$

where $\sigma^{a}, a=1,2,3$, are either the left-invariant $\sigma_{R}^{a}$ or the right-invariant $\sigma_{L}^{a}$ one-forms on the three-sphere: $d \sigma^{a}=\frac{1}{2} \eta \epsilon^{a}{ }_{b c} \sigma^{b} \wedge \sigma^{c}$ with $\eta=1$ if $\sigma=\sigma_{R}$ and $\eta=-1$ if $\sigma=\sigma_{L}$. We can then take the vierbein

$$
\begin{aligned}
\tilde{e}^{4} & =d r \\
\tilde{e}^{a} & =\frac{r}{2} \sigma^{a}
\end{aligned}
$$

If we take the hyper-Kähler structure (in Cartesian coordinates) given by

$$
\begin{aligned}
& J^{1}=-\left(d x^{1} \wedge d x^{3}+d x^{2} \wedge d x^{4}\right), \\
& J^{2}=-\left(d x^{1} \wedge d x^{2}-d x^{3} \wedge d x^{4}\right), \\
& J^{3}=-\left(d x^{1} \wedge d x^{4}-d x^{2} \wedge d x^{3}\right),
\end{aligned}
$$

we have that $\psi=0$. See [37] for the coordinate transformation to express these forms in terms of $\sigma^{a}$. Requiring the two-forms (4.10) to be anti-self-dual imposes the orientation $\tilde{e}^{4} \wedge \tilde{e}^{1} \wedge \tilde{e}^{2} \wedge \tilde{e}^{3}$. For the simple case that $j^{\alpha}$ and $H$ only depend on $u$ and $r$, we can write the harmonic functions

$$
j^{\alpha} H=P^{\alpha}(u)+\frac{Q^{\alpha}(u)}{r^{2}},
$$

where $P^{\alpha}, Q^{\alpha}$ are arbitrary functions of $u$ that will be fixed by the other equations of motion. From $j_{\alpha} j^{\alpha}=1$ we find

$$
H=\sqrt{\Omega_{\alpha \beta}\left(P^{\alpha}+\frac{Q^{\alpha}}{r^{2}}\right)\left(P^{\beta}+\frac{Q^{\beta}}{r^{2}}\right)} .
$$


Notice that (4.4) reduces to

$$
\tilde{d} \mathcal{G}^{+\alpha}=\mathcal{L}_{\partial_{u} \star_{4}} \tilde{d}\left(j^{\alpha} H\right) .
$$

Using the self-duality of $\mathcal{G}^{+\alpha}$ we can write

$$
\mathcal{G}^{+\alpha}=C_{b}^{\alpha} \tilde{e}^{4} \wedge \tilde{e}^{b}+\frac{1}{2} C_{b}^{\alpha} \epsilon_{c d}^{b} \tilde{e}^{c} \wedge \tilde{e}^{d},
$$

where we assume that $C_{b}^{\alpha}$ only depend on $u$ and $r$ (to stay in line with [7]). We can calculate

$$
\tilde{d} \mathcal{G}^{+\alpha}=\frac{1}{r^{2}}\left[(1-\eta) r C_{b}^{\alpha}+\frac{1}{2} r^{2} \partial_{r}\left(C_{b}^{\alpha}\right)\right] \epsilon_{c d}^{b} \tilde{e}^{4} \wedge \tilde{e}^{c} \wedge \tilde{e}^{d} .
$$

Substitution in (4.13) yields

$$
\begin{aligned}
\frac{1}{r^{2}}\left[(1-\eta) r C_{b}^{\alpha}+\frac{1}{2} r^{2} \partial_{r}\left(C_{b}^{\alpha}\right)\right] \epsilon_{c d}^{b} \tilde{e}^{4} \wedge \tilde{e}^{c} \wedge \tilde{e}^{d} & =\mathcal{L}_{\partial_{u} \star} \star_{4} \tilde{d}\left(j^{\alpha} H\right) \\
& =-2 \frac{\partial_{u} Q^{\alpha}}{r^{3}} \tilde{e}^{1} \wedge \tilde{e}^{2} \wedge \tilde{e}^{3}
\end{aligned}
$$

from which it follows that

$$
\begin{aligned}
\partial_{u} Q^{\alpha} & =0, \\
\partial_{r}\left(C_{b}^{\alpha}\right) & =2(\eta-1) \frac{1}{r} C_{b}^{\alpha} .
\end{aligned}
$$

The second equation is solved by

$$
C_{b}^{\alpha}=C_{b}^{\alpha}(u) r^{2(\eta-1)},
$$

for functions $C_{b}^{\alpha}(u)$. Hence

$$
\mathcal{G}^{+\alpha}=C_{b}^{\alpha}(u) r^{2(\eta-1)} \tilde{e}^{4} \wedge \tilde{e}^{b}+\frac{1}{2} C_{b}^{\alpha}(u) r^{2(\eta-1)} \epsilon_{c d}^{b} \tilde{e}^{c} \wedge \tilde{e}^{d} .
$$

Assuming as in [7] that

$$
\omega=W(u, r) \sigma^{3},
$$

we can calculate that

$$
(\tilde{d} \omega)^{+}=\left(\frac{2}{r^{2}} \eta W+\frac{1}{r} \partial_{r} W\right)\left(\tilde{e}^{1} \wedge \tilde{e}^{2}-\tilde{e}^{3} \wedge \tilde{e}^{4}\right) .
$$

We then find from substitution of (4.11), (4.19) and (4.21) in

$$
(\tilde{d} \omega)^{+}=H j_{\alpha} \mathcal{G}^{+\alpha}
$$

that

$$
\begin{aligned}
C_{1}^{\alpha}(u) & =C_{2}^{\alpha}(u)=0, \\
W & =W_{1}(u) r^{-2 \eta}+\frac{1}{2} \Omega_{\alpha \beta} C_{3}^{\alpha}(u)\left(\frac{P^{\beta}}{2 \eta} r^{2 \eta}+\frac{Q^{\beta}}{2 \eta-1} r^{2 \eta-2}\right),
\end{aligned}
$$


where $W_{1}$ is yet another arbitrary function of $u$. We then find from (4.11), (4.19) and (4.22) that

$$
\begin{aligned}
H_{\mathrm{SD}}^{\alpha} & =H \mathcal{G}^{+\alpha}-j^{\alpha}(\tilde{d} \omega)^{+} \\
& =\left[H C_{3}^{\alpha}(u) r^{2(\eta-1)}-j^{\alpha} C_{3}^{\beta}(u) r^{2(\eta-1)} \Omega_{\beta \gamma}\left(P^{\gamma}(u)+\frac{Q^{\gamma}(u)}{r^{2}}\right)\right]\left(\tilde{e}^{1} \wedge \tilde{e}^{2}-\tilde{e}^{3} \wedge \tilde{e}^{4}\right) .
\end{aligned}
$$

Lastly, the Einstein equation (4.7) reduces to

$$
\begin{aligned}
\tilde{\nabla}^{i}(\dot{\omega})_{i}-\frac{1}{2} \tilde{\nabla}^{2} \mathcal{F}= & 2 H \partial_{u}^{2}(H)+\partial_{u}(H) \partial_{u}(H)-\frac{1}{2} H^{-2}\left((\tilde{d} \omega)^{+}\right)^{2} \\
& -\frac{1}{2} H^{-2} \Omega_{\alpha \beta} H_{\mathrm{SD}}^{\alpha} \cdot H_{\mathrm{SD}}^{\beta}-\Omega_{\alpha \beta} H^{2} \partial_{u} j^{\alpha} \partial_{u} j^{\beta} .
\end{aligned}
$$

Using (4.11), (4.12), (4.20), (4.23) and (4.24), and assuming that $\mathcal{F}=\mathcal{F}(u, r)$ we derive that

$$
\partial_{r}\left(r^{3} \partial_{r} \mathcal{F}\right)=-2 \Omega_{\alpha \beta} \dot{P}^{\alpha} \dot{P}^{\beta} r^{3}-4 \Omega_{\alpha \beta}\left(P^{\alpha} r^{3}+Q^{\alpha} r\right) \partial_{u}^{2} P^{\beta}+2 \Omega_{\alpha \beta} C_{3}^{\alpha}(u) C_{3}^{\beta}(u) r^{4 \eta-1} .
$$

Integration of this equation yields

$$
\begin{aligned}
\mathcal{F}= & C_{5}(u)-\frac{1}{2} C_{4}(u) \frac{1}{r^{2}}-\frac{1}{2} \Omega_{\alpha \beta}\left(P^{\alpha} \partial_{u}^{2} P^{\beta}+\frac{1}{2} \dot{P}^{\alpha} \dot{P}^{\beta}\right) r^{2} \\
& +\frac{1}{4 \eta(2 \eta-1)} \Omega_{\alpha \beta} C_{3}^{\alpha}(u) C_{3}^{\beta}(u) r^{4 \eta-2}-2 \Omega_{\alpha \beta} Q^{\alpha} \partial_{u}^{2} P^{\beta} \log (r),
\end{aligned}
$$

for arbitrary functions $C_{4}$ and $C_{5}$. This construction can easily be extended to other hyper-Kähler base spaces.

\section{$4.2 u$-independent solutions}

A second class of solutions in which the general equations simplify considerably is the class that does not depend on $u$. We can see this as introducing an extra symmetry of the solution. In particular we get an extra Killing vector $\partial_{u}$, which is spacelike when $\mathcal{F}>0$ and timelike when $\mathcal{F}<0$. The three-forms reduce to

$$
\hat{G}^{\alpha}=-H e^{+} \wedge e^{-} \wedge \tilde{d}\left(H^{-1} j^{\alpha}\right)+j^{\alpha} H^{-1} e^{+} \wedge \tilde{d} \beta+e^{-} \wedge\left[-j^{\alpha}(\tilde{d} \omega)^{-}+H_{\mathrm{SD}}^{\alpha}\right]+\star_{4} \tilde{d}\left(j^{\alpha} H\right) .
$$

The base space has to be hyper-Kähler since from (3.17)

$$
\tilde{d} J^{a}=0
$$

and $\beta$ has self-dual curvature:

$$
\tilde{d} \beta=\star_{4} \tilde{d} \beta .
$$

The Bianchi identity, (3.18) and (3.19), and Einstein equation (3.24) reduce to respectively

$$
\begin{aligned}
\tilde{d} \mathcal{G}^{+\alpha} & =0, \\
\tilde{d} \star_{4} \tilde{d}\left(j^{\alpha} H\right) & =-\tilde{d} \beta \wedge \mathcal{G}^{+\alpha}, \\
\tilde{\nabla}^{2} \mathcal{F} & =\Omega_{\alpha \beta} \mathcal{G}^{+\alpha} \cdot \mathcal{G}^{+\beta},
\end{aligned}
$$


where

$$
\mathcal{G}^{+\alpha}=H^{-1}\left[j^{\alpha}(\tilde{d} \omega)^{+}+\frac{1}{2} j^{\alpha} \mathcal{F} \tilde{d} \beta+H_{\mathrm{SD}}^{\alpha}\right],
$$

and we have used that

$$
\Omega_{\alpha \beta} \mathcal{G}^{+\alpha} \cdot \mathcal{G}^{+\beta}=H^{-2}\left[(\tilde{d} \omega)^{+}+\frac{1}{2} \mathcal{F} \tilde{d} \beta\right] \cdot\left[(\tilde{d} \omega)^{+}+\frac{1}{2} \mathcal{F} \tilde{d} \beta\right]-H^{-2} g_{\alpha \beta} H_{\mathrm{SD}}^{\alpha} \cdot H_{\mathrm{SD}}^{\beta} .
$$

When the Killing vector $\partial_{u}$ is spacelike, the $u$-direction can be compactified on a circle and we can reduce the solution to five dimensions. This will be done in the next section. In section 4.4 we then take the base space $\mathcal{B}$ to be Gibbons-Hawking [42] which has yet another Killing vector. Assuming that this symmetry extends to the whole solution, we solve the equations of motion completely.

\subsection{Reduction to five dimensions}

When one considers a $u$-independent solution with $\mathcal{F}$ positive such that $\partial_{u}$ is a spacelike Killing vector, one can compactify this direction on a circle and do a Kaluza-Klein reduction to obtain a five-dimensional solution. The six-dimensional metric reduces to the fivedimensional metric $d s_{5}^{2}$, a Kaluza-Klein vector $A^{0}$ and a scalar $X^{0}$. The three-forms $\hat{G}^{\alpha}$ reduce to three-forms $G^{\alpha}$ and two-forms $F^{\alpha}$ that are related to each other since the $\hat{G}^{\alpha}$ obey a self-duality condition. The scalars $j^{\alpha}$ reduce to scalars $X^{\alpha}$. Reducing the six-dimensional theory to five-dimensions thus results in five-dimensional supergravity coupled to $n_{T}+1$ vector multiplets. We can express the six-dimensional data in terms of five-dimensional data by [29]

$$
\begin{aligned}
d s_{6}^{2} & =r^{2}\left(d u+A^{0}\right)^{2}+r^{-2 / 3} d s_{5}^{2}, \\
\hat{G}^{\alpha} & =G^{\alpha}-F^{\alpha} \wedge\left(d u+A^{0}\right), \\
j^{\alpha} & =r^{-2 / 3} X^{\alpha}, \\
r^{-4 / 3} & =X^{0},
\end{aligned}
$$

where

$$
\begin{aligned}
d s_{5}^{2} & =-f^{2}(d v+\omega)^{2}+f^{-1} d s_{4}^{2}, \\
G^{\alpha} & =d B^{\alpha}+A^{\alpha} \wedge F^{0},
\end{aligned}
$$

for a function $f$ and two-forms $B^{\alpha}$. The scalars $X^{I}, I \in\left\{0,1, \ldots, n_{T}+1\right\}$, are the so-called very special coordinates. These are $n_{T}+2$ real coordinates that describe an $n_{T}+2$-dimensional manifold in which the scalar manifold is given by the hypersurface [58]

$$
\mathcal{N} \equiv \frac{1}{3 !} C_{I J K} X^{I} X^{J} X^{K}=1
$$

where $C_{I J K}$ is a constant symmetric tensor and $\mathcal{N}$ is the so-called cubic potential. The potential $\mathcal{N}$ in terms of six-dimensional data is given by [29]

$$
\mathcal{N}=\Omega_{\alpha \beta} X^{0} X^{\alpha} X^{\beta} .
$$


It is straightforward to derive that

$$
\begin{aligned}
d s_{5}^{2} & =-\left(\mathcal{F} H^{2}\right)^{-2 / 3}(d v+\omega)^{2}+\left(\mathcal{F} H^{2}\right)^{1 / 3} d s_{4}^{2}, \\
X^{0} & =\left(\mathcal{F} H^{-1}\right)^{-2 / 3}, \\
X^{\alpha} & =\left(\mathcal{F} H^{-1}\right)^{1 / 3} j^{\alpha}, \\
F^{0} & =\tilde{d}\left[\beta+\mathcal{F}^{-1}(d v+\omega)\right], \\
F^{\alpha} & =\tilde{d}\left[H^{-1} j^{\alpha}(d v+\omega)\right]-H^{-1}\left[j^{\alpha}(\tilde{d} \omega)^{+}+\frac{1}{2} j^{\alpha} \mathcal{F} \tilde{d} \beta+H_{\mathrm{SD}}^{\alpha}\right] .
\end{aligned}
$$

Note that the field strengths can be written as

$$
F^{I}=\tilde{d}\left[X^{I} f(d v+\omega)\right]+\Theta^{I},
$$

where

$$
\Theta^{0}=\tilde{d} \beta, \quad \Theta^{\alpha}=-\mathcal{G}^{\alpha},
$$

are self-dual tensors. Also (4.31) implies that the two-forms $\Theta^{I}$ are closed. Using

$$
X_{I} \equiv \frac{1}{6} C_{I J K} X^{J} X^{K}
$$

we find that

$$
X_{I} \Theta^{I}=-\frac{2}{3} f(\tilde{d} \omega)^{+} .
$$

Furthermore, we find that (4.32) reduces to

$$
\tilde{\nabla}^{2}\left(f^{-1} X_{\alpha}\right)=\frac{1}{6} C_{\alpha J K} \Theta^{J} \cdot \Theta^{K},
$$

and that the Einstein equation (4.33) reduces to

$$
\tilde{\nabla}^{2}\left(f^{-1} X_{0}\right)=\frac{1}{6} C_{0 J K} \Theta^{J} \cdot \Theta^{K} .
$$

This implies that we find ourselves exactly in the timelike class of five-dimensional solutions of $[39,59]$. Thus every solution of five-dimensional supergravity coupled to an arbitrary number of vector multiplets in the timelike class that has a cubic potential of the form

$$
\mathcal{N}=\frac{1}{3 !} C_{I J K} X^{I} X^{J} X^{K}=\Omega_{\alpha \beta} X^{0} X^{\alpha} X^{\beta},
$$

can be uplifted to six dimensions. An interesting remark is that classical $M$-theory solutions do not have cubic potentials of this form. To lift them up, one also has to take into account the one-loop contributions coming from the reduction on the circle $[29,30]$.

Minimal five-dimensional supergravity. After the reduction we always end up with at least one vector multiplet in five dimensions. However, one can truncate the reduction of minimal supergravity in six dimensions to minimal five-dimensional supergravity [7]. We get minimal supergravity when we set the three-forms of the tensor multiplets $H^{M}=0$ for $M=1, \ldots, n_{T}$ and furthermore set $j^{\alpha}=0$ for $\alpha=2, \ldots, n_{T}+1$ and $j^{1}=1$. The 
only three-form that is non-zero is $\hat{G}^{1}$. We can truncate the reduced theory to minimal supergravity by getting rid of the scalars, which can be done by setting $\mathcal{F}=H$ such that $X^{0}=X^{1}=1$. Consistency of (4.32) and (4.33) then enforces $\tilde{d} \beta=-\mathcal{G}^{+1}$ or

$$
\tilde{d} \beta=-\frac{2}{3} H^{-1}(\tilde{d} \omega)^{+} .
$$

This implies that

$$
F \equiv F^{0}=F^{1}=\tilde{d}\left[\beta+\mathcal{F}^{-1}(d v+\omega)\right] .
$$

Introducing $G^{+} \equiv f(\tilde{d} \omega)^{+}$, we find that

$$
\tilde{d} G^{+}=0
$$

and that (4.47) reduces to

$$
\tilde{\nabla}^{2}\left(f^{-1}\right)=\frac{4}{9} G^{+} \cdot G^{+}
$$

This means that we find ourselves in the timelike class of minimal five-dimensional supergravity [37]. ${ }^{3}$

The null class of minimal five-dimensional supergravity arises from reducing nontwisting solutions of minimal six-dimensional supergravity that have a Gibbons-Hawking base space [7].

\subsection{Gibbons-Hawking base space}

We now consider $u$-independent solutions with a Gibbons-Hawking base space [42]. This is the most general hyper-Kähler four-manifold admitting a Killing vector field $\partial_{\psi},{ }^{4}$ preserving the three complex structures [60]. It has the metric

$$
d s_{4}^{2}=H_{2}^{-1}\left(d \psi+\chi_{a} d x^{a}\right)^{2}+H_{2} \delta_{a b} d x^{a} d x^{b}
$$

where $a=1,2,3, \chi_{a}$ and $H_{2}$ are independent of $\psi$ and

$$
\begin{aligned}
\nabla^{2} H_{2} & =0, \\
\vec{\nabla} \times \vec{\chi} & =\vec{\nabla} H_{2} .
\end{aligned}
$$

We take $\nabla$ with respect to three-dimensional flat space.

We now obtain all solutions in the case the symmetry $\partial_{\psi}$ of the base space is extended to a symmetry of the full solution. This was done in [37] for minimal five-dimensional supergravity, in [7] for minimal six-dimensional supergravity and in [59] for five-dimensional supergravity coupled to an arbitrary number of vector multiplets, so we will be quite brief here.

\footnotetext{
${ }^{3}$ They use a different normalization of the field strength: $F_{\text {here }}=\frac{2}{\sqrt{3}} F_{\text {there }}$.

${ }^{4}$ Notice that this $\psi$ is not related to the $\psi$ in terms of the complex structures. We can safely do this since from (3.14) we see that $\psi=0$ for this class of solutions.
} 
We can choose the vierbein

$$
\begin{aligned}
& \tilde{e}^{4}=H_{2}^{-1 / 2}\left(d \psi+\chi_{a} d x^{a}\right), \\
& \tilde{e}^{a}=H_{2}^{1 / 2} d x^{a} .
\end{aligned}
$$

Anti-self-duality of the complex structure forms implies that the volume form is given by $\tilde{e}^{4} \wedge \tilde{e}^{1} \wedge \tilde{e}^{2} \wedge \tilde{e}^{3}$. We can decompose the one-forms

$$
\begin{aligned}
& \beta=\beta_{0}\left(d \psi+\chi_{a} d x^{a}\right)+\beta_{a} d x^{a}, \\
& \omega=\omega_{0}\left(d \psi+\chi_{a} d x^{a}\right)+\omega_{a} d x^{a},
\end{aligned}
$$

where $\beta_{0}, \beta_{a}, \omega_{0}$ and $\omega_{a}$ are functions on $\mathbb{R}^{3}$. Solving (4.30) results in

$$
\beta_{0}=H_{2}^{-1} H_{3}, \quad \vec{\nabla} \times \vec{\beta}=-\vec{\nabla} H_{3},
$$

with $H_{3}$ an arbitrary harmonic function on $\mathbb{R}^{3}$. The two-form $\mathcal{G}^{+\alpha}$ is self-dual so it has to be of the form

$$
\mathcal{G}^{+\alpha}=-\frac{1}{2} C_{b}^{\alpha} \tilde{e}^{4} \wedge \tilde{e}^{b}-\frac{1}{4} C_{b}^{\alpha} \epsilon_{c d}^{b} \tilde{e}^{c} \wedge \tilde{e}^{d},
$$

and solving (4.31) results in

$$
\vec{C}^{\alpha}=2 \vec{\nabla}\left(H_{2}^{-1} H_{4}^{\alpha}\right),
$$

with $H_{4}^{\alpha}$ arbitrary harmonic functions on $\mathbb{R}^{3}$. Using this result one can solve (4.32), which results in

$$
j^{\alpha} H=H_{1}^{\alpha}-H_{2}^{-1} H_{3} H_{4}^{\alpha},
$$

where $H_{1}^{\alpha}$ are arbitrary harmonic functions on $\mathbb{R}^{3}$. Using $j_{\alpha} j^{\alpha}=1$ we find

$$
H=\sqrt{\Omega_{\alpha \beta}\left(H_{1}^{\alpha}-H_{2}^{-1} H_{3} H_{4}^{\alpha}\right)\left(H_{1}^{\beta}-H_{2}^{-1} H_{3} H_{4}^{\beta}\right)} .
$$

With the solution of $\mathcal{G}^{+\alpha},(4.33)$ can be solved and yields

$$
\mathcal{F}=-H_{5}+H_{2}^{-1} \Omega_{\alpha \beta} H_{4}^{\alpha} H_{4}^{\beta},
$$

with $H_{5}$ an arbitrary harmonic function on $\mathbb{R}^{3}$. Now, using that

$$
\begin{aligned}
j_{\alpha} \mathcal{G}^{+\alpha} & =-j_{\alpha} \nabla_{b}\left(H_{2}^{-1} H_{4}^{\alpha}\right) \tilde{e}^{4} \wedge \tilde{e}^{b}-\frac{1}{2} j_{\alpha} \nabla_{b}\left(H_{2}^{-1} H_{4}^{\alpha}\right) \epsilon_{c d}^{b} \tilde{e}^{c} \wedge \tilde{e}^{d} \\
& =H^{-1}(\tilde{d} \omega)^{+}+\frac{1}{2} \mathcal{F} H^{-1} \tilde{d} \beta
\end{aligned}
$$

we get an equation for $\omega$ :

$$
\begin{aligned}
H_{2} \vec{\nabla} \omega_{0}-\omega_{0} \vec{\nabla} H_{2}-\vec{\nabla} \times \vec{\omega}= & 2 \Omega_{\alpha \beta}\left(H_{1}^{\alpha} H_{2}-H_{3} H_{4}^{\alpha}\right) \vec{\nabla}\left(H_{2}^{-1} H_{4}^{\beta}\right) \\
& +\left(H_{5} H_{2}-\Omega_{\alpha \beta} H_{4}^{\alpha} H_{4}^{\beta}\right) \vec{\nabla}\left(H_{2}^{-1} H_{3}\right) .
\end{aligned}
$$

Taking the divergence of this equation yields an integrability equation that can be solved for $\omega_{0}$ :

$$
\omega_{0}=H_{6}-H_{2}^{-2} H_{3} \Omega_{\alpha \beta} H_{4}^{\alpha} H_{4}^{\beta}+H_{2}^{-1} \Omega_{\alpha \beta} H_{1}^{\alpha} H_{4}^{\beta}+\frac{1}{2} H_{2}^{-1} H_{3} H_{5},
$$


with $H_{6}$ an arbitrary harmonic function on $\mathbb{R}^{3}$. Substitution of this in (4.64) gives an equation that determines $\vec{\omega}$ up to a gradient (and this can be eliminated by shifting $v$ ):

$$
\vec{\nabla} \times \vec{\omega}=\Omega_{\alpha \beta}\left(H_{4}^{\alpha} \vec{\nabla} H_{1}^{\beta}-H_{1}^{\alpha} \vec{\nabla} H_{4}^{\beta}\right)+H_{2} \vec{\nabla} H_{6}-H_{6} \vec{\nabla} H_{2}+\frac{1}{2} H_{3} \vec{\nabla} H_{5}-\frac{1}{2} H_{5} \vec{\nabla} H_{3} .
$$

From the definition of $\mathcal{G}^{+\alpha}$ we then find that

$$
H_{\mathrm{SD}}^{\alpha}=-D_{b}^{\alpha} \tilde{e}^{4} \wedge \tilde{e}^{b}-\frac{1}{2} D_{b}^{\alpha} \epsilon_{c d}^{b} \tilde{e}^{c} \wedge \tilde{e}^{d},
$$

where

$$
\vec{D}^{\alpha} \equiv H \vec{\nabla}\left(H_{2}^{-1} H_{4}^{\alpha}\right)-j^{\alpha} H j_{\beta} \vec{\nabla}\left(H_{2}^{-1} H_{4}^{\beta}\right) .
$$

We now consider the so-called multi-centered Gibbons-Hawking subclass of these solutions. We introduce the notation

$$
\begin{aligned}
\mathbb{H} & \equiv\left(H_{1}^{\alpha}, H_{2}, H_{3}, H_{4}^{\alpha}, H_{5}, H_{6}\right), \\
\Gamma_{A} & \equiv\left(\mu_{A}^{\alpha}, m_{A}, q_{A}, p_{A}^{\alpha}, n_{A}, j_{A}\right), \\
\Gamma_{\infty} & \equiv\left(\mu_{\infty}^{\alpha}, m_{\infty}, q_{\infty}, p_{\infty}^{\alpha}, n_{\infty}, j_{\infty}\right),
\end{aligned}
$$

where $A=1, \ldots, N$ and all the components of the vectors $\Gamma_{A}$ and $\Gamma_{\infty}$ are constants. We then take the harmonic functions of the form

$$
\mathbb{H}=\Gamma_{\infty}+\sum_{A} \frac{\Gamma_{A}}{\left|\vec{x}-\vec{x}_{A}\right|}
$$

Although every set of centers $\vec{x}_{A}$ describes a solution, there will typically be Dirac stringlike singularities. Imposing the absence of these singularities gives a constraint on the relative positions, see $[34,61,62]$. This arises from requiring $\vec{\omega}$ to be globally defined, which implies that $d^{2} \vec{\omega}=0$. If we define the symplectic product \langle\rangle working on vectors of the form $v=\left(v_{1}^{\alpha}, v_{2}, v_{3}, v_{4}^{\alpha}, v_{5}, v_{6}\right)$ via

$$
\langle v, w\rangle=\Omega_{\alpha \beta} v_{4}^{\alpha} w_{1}^{\beta}-\Omega_{\alpha \beta} v_{1}^{\alpha} w_{4}^{\beta}+v_{2} w_{6}-v_{6} w_{2}+\frac{1}{2}\left(v_{3} w_{5}-v_{5} w_{3}\right),
$$

we can rewrite (4.66) as

$$
\star_{3} d \vec{\omega}=\langle\mathbb{H}, d \mathbb{H}\rangle .
$$

Taking $d \star_{3}$ on both sides leads to

$$
\sum_{B \neq A} \frac{\left\langle\Gamma_{A}, \Gamma_{B}\right\rangle}{\left|\vec{x}_{A}-\vec{x}_{B}\right|}=\left\langle\Gamma_{\infty}, \Gamma_{A}\right\rangle, \quad A=1, \ldots, N .
$$

These are usually referred to as "Bubble equations" since they control the size of the two-cycles or bubbles in the Gibbons-Hawking base space [63].

Let $\operatorname{Sp}\left(6+2 n_{T}, \mathbb{R}\right)$ denote the symplectic group that preserves the symplectic product \langle\rangle . A linear combination of harmonic functions is still harmonic, hence sending $\mathbb{H} \rightarrow g \mathbb{H}$ with $g \in \mathrm{Sp}\left(6+2 n_{T}, \mathbb{R}\right)$ sends a solution to a solution, preserving regularity. This symplectic group was earlier noticed for minimal supergravity in [43]. 
Summary. The most general $u$-independent solution with a Gibbons-Hawking base space whose Killing vector field extends to a symmetry of the full solution is determined by $6+2 n_{T}$ harmonic functions $H_{1}^{\alpha}, H_{2}, H_{3}, H_{4}^{\alpha}, H_{5}$ and $H_{6}$ on $\mathbb{R}^{3}$. Its metric is given by

$$
\begin{aligned}
& d s_{6}^{2}=2 H^{-1}(d u+\beta)\left(d v+\omega+\frac{1}{2} \mathcal{F}(d u+\beta)\right)+H d s_{4}^{2}, \\
& d s_{4}^{2}=H_{2}^{-1}\left(d \psi+\chi_{a} d x^{a}\right)^{2}+H_{2} \delta_{a b} d x^{a} d x^{b},
\end{aligned}
$$

where

$$
\begin{aligned}
\vec{\nabla} \times \vec{\chi} & =\vec{\nabla} H_{2}, \\
H & =\sqrt{\Omega_{\alpha \beta}\left(H_{1}^{\alpha}-H_{2}^{-1} H_{3} H_{4}^{\alpha}\right)\left(H_{1}^{\beta}-H_{2}^{-1} H_{3} H_{4}^{\beta}\right)}, \\
\mathcal{F} & =-H_{5}+H_{2}^{-1} \Omega_{\alpha \beta} H_{4}^{\alpha} H_{4}^{\beta} .
\end{aligned}
$$

The one-forms are decomposed as

$$
\begin{aligned}
& \beta=\beta_{0}\left(d \psi+\chi_{a} d x^{a}\right)+\beta_{a} d x^{a}, \\
& \omega=\omega_{0}\left(d \psi+\chi_{a} d x^{a}\right)+\omega_{a} d x^{a},
\end{aligned}
$$

with the coefficients $\beta_{0}, \beta_{a}, \omega_{0}$ and $\omega_{a}$ given by

$$
\begin{aligned}
\beta_{0} & =H_{2}^{-1} H_{3}, \\
\vec{\nabla} \times \vec{\beta} & =-\vec{\nabla} H_{3}, \\
\omega_{0} & =H_{6}-H_{2}^{-2} H_{3} \Omega_{\alpha \beta} H_{4}^{\alpha} H_{4}^{\beta}+H_{2}^{-1} \Omega_{\alpha \beta} H_{1}^{\alpha} H_{4}^{\beta}+\frac{1}{2} H_{2}^{-1} H_{3} H_{5}, \\
\vec{\nabla} \times \vec{\omega} & =\Omega_{\alpha \beta}\left(H_{4}^{\alpha} \vec{\nabla} H_{1}^{\beta}-H_{1}^{\alpha} \vec{\nabla} H_{4}^{\beta}\right)+H_{2} \vec{\nabla} H_{6}-H_{6} \vec{\nabla} H_{2}+\frac{1}{2} H_{3} \vec{\nabla} H_{5}-\frac{1}{2} H_{5} \vec{\nabla} H_{3} .
\end{aligned}
$$

The three-forms are equal to

$$
\hat{G}^{\alpha}=-H e^{+} \wedge e^{-} \wedge \tilde{d}\left(H^{-1} j^{\alpha}\right)+j^{\alpha} H^{-1} e^{+} \wedge \tilde{d} \beta+e^{-} \wedge\left[-j^{\alpha}(\tilde{d} \omega)^{-}+H_{\mathrm{SD}}^{\alpha}\right]+\star_{4} \tilde{d}\left(j^{\alpha} H\right),
$$

where

$$
\begin{aligned}
e^{-} & =H^{-1}(d u+\beta), \\
e^{+} & =d v+\omega+\frac{1}{2} \mathcal{F} H e^{-}, \\
H_{\mathrm{SD}}^{\alpha} & =-D_{b}^{\alpha}\left(d \psi+\chi_{a} d x^{a}\right) \wedge d x^{b}-\frac{1}{2} H_{2} D_{b}^{\alpha} \epsilon_{c d}^{b} d x^{c} \wedge d x^{d}, \\
\vec{D}^{\alpha} & =H \vec{\nabla}\left(H_{2}^{-1} H_{4}^{\alpha}\right)-j^{\alpha} H j_{\beta} \vec{\nabla}\left(H_{2}^{-1} H_{4}^{\beta}\right) .
\end{aligned}
$$

Lastly, the scalars are given by

$$
j^{\alpha}=\frac{H_{1}^{\alpha}-H_{2}^{-1} H_{3} H_{4}^{\alpha}}{H} .
$$

When the harmonic functions are taken of the form (4.70), one has to impose the bubble equations (4.73) in order to avoid Dirac string-like singularities. 


\subsection{Black strings and other objects with a horizon}

In [8] it is shown that in supergravity coupled to tensor multiplets, near horizon geometries of black objects are locally either $\mathbb{R}^{1,1} \times T^{4}, \mathbb{R}^{1,1} \times K_{3}$ or $A d S_{3} \times S^{3}$. In this section we will consider some examples of the latter local geometry which can correspond to a black string or the uplift of a black ring or black lens.

Black string. When taking a solution of section 4.4, compactifying the $u$-direction on a circle, taking the harmonic functions of the form (4.70) with only one center $\vec{x}_{1}=0$ and requiring the metric to asymptote to $\mathbb{R} \times S^{1} \times \mathbb{R}^{4} / \mathbb{Z}_{m}$, we find a generalization of the single black string solution in [43]. Perhaps the most interesting case is $m=1$, which at infinity corresponds to a black string wrapped around a circle times a flat $5 \mathrm{~d}$ Minkowski spacetime. For $m \neq 1$ one gets ALE spaces.

The string is wound around the $u$-direction and becomes a black hole after reduction on the $u$-circle. In appendix $\mathrm{D}$ we derive that to get the right asymptotics for the metric, we need

$$
\Gamma_{\infty}=\left(\mu_{\infty}^{\alpha}, 0,0,0,-1, \frac{1}{2} \frac{q}{m}-\frac{1}{m} \Omega_{\alpha \beta} \mu_{\infty}^{\alpha} p^{\beta}\right),
$$

with

$$
\Omega_{\alpha \beta} \mu_{\infty}^{\alpha} \mu_{\infty}^{\beta}=1
$$

Using spherical coordinates $r, \theta, \phi$ for the $\mathbb{R}^{3}$ part, the metric of the solution is then given by

$$
\begin{aligned}
d s_{6}^{2}= & 2\left(1+2 \frac{\Omega_{\alpha \beta} \mu_{\infty}^{\alpha} \tilde{Q}^{\beta}}{4 \sqrt{2} r}+\frac{\Omega_{\alpha \beta} \tilde{Q}^{\alpha} \tilde{Q}^{\beta}}{32 r^{2}}\right)^{-1 / 2} d u^{\prime} \\
& \times\left[d v+\frac{J_{\psi}}{8 r}(d \psi+m \cos (\theta) d \phi)+\frac{1}{2}\left(1+\frac{Q}{4 r}\right) d u^{\prime}\right] \\
& +\left(1+2 \frac{\Omega_{\alpha \beta} \mu_{\infty}^{\alpha} \tilde{Q}^{\beta}}{4 \sqrt{2} r}+\frac{\Omega_{\alpha \beta} \tilde{Q}^{\alpha} \tilde{Q}^{\beta}}{32 r^{2}}\right)^{1 / 2}\left[\frac{r}{m}(d \psi+m \cos (\theta) d \phi)^{2}+\frac{m}{r} d r^{2}+m r d \Omega_{2}^{2}\right],
\end{aligned}
$$

where we defined $u^{\prime}=u+\frac{q}{m} \psi$. To make this transformation well-defined, we have to impose

$$
\frac{4 \pi q}{l m} \in \mathbb{Z}
$$

where $l$ is the length of the $u$-circle. We also defined

$$
\begin{aligned}
\tilde{Q}^{\alpha} & \equiv 4 \sqrt{2}\left(\mu^{\alpha}-\frac{1}{m} q p^{\alpha}\right), \\
Q & \equiv 4\left(-n+\frac{1}{m} \Omega_{\alpha \beta} p^{\alpha} p^{\beta}\right), \\
J_{\psi} & \equiv 8\left(j-\frac{1}{m^{2}} q \Omega_{\alpha \beta} p^{\alpha} p^{\beta}+\frac{1}{m}\left(\Omega_{\alpha \beta} \mu^{\alpha} p^{\beta}+\frac{1}{2} q n\right)\right) .
\end{aligned}
$$


The near horizon geometry of this solution is a direct product of an extremal BTZ black hole and a round $S^{3} / \mathbb{Z}_{m}$. The entropy of the black string is given by

$$
S=\frac{A}{4 G_{\mathrm{N}}^{(6)}}=2 \pi \sqrt{\frac{1}{2} m Q \Omega_{\alpha \beta} \tilde{Q}^{\alpha} \tilde{Q}^{\beta}-J_{\psi}^{2}},
$$

where we used conventions in which $G_{\mathrm{N}}^{(6)}=\frac{l \pi}{4}$.

These black holes can be embedded in F-theory and we consider the case $m=1$ for simplicity. We take an F-theory background $\mathbb{R} \times S^{1} \times \mathbb{R}^{4} \times C Y_{3}$, where $C Y_{3}$ is a smooth elliptically fibered Calabi-Yau three-fold $\pi: C Y_{3} \rightarrow B_{2}$. The solution corresponds to a D3-brane wrapped on $S^{1} \times C$, where $C \subset B_{2}$ is a curve. We have the set of vertical divisors $D_{\alpha} \equiv \pi^{-1}\left(D_{\alpha}^{b}\right)$, where $D_{\alpha}^{b}$ are divisors of $B_{2}$ that are chosen such that

$$
\Omega_{\alpha \beta}=\int_{B_{2}} \omega_{\alpha} \wedge \omega_{\beta}
$$

for $\omega_{\alpha}$ the two-form classes Poincare dual to $D_{\alpha}$. We can then write $C=q^{\alpha} \omega_{\alpha}$ for the form Poincare dual to the curve $C$. The strings that one gets after compactification on $C Y_{3}$ carry $n$ units of momentum along the circle. There is also an $\mathrm{SO}(4) \equiv \mathrm{SU}(2)_{L} \times \mathrm{SU}(2)_{R}$ symmetry from rotations in the $\mathbb{R}^{4}$ plane transverse to the D3-brane. The entropy corresponding to a single string to leading order in the large charge limit is given by [22]

$$
S=2 \pi \sqrt{\frac{1}{2} n \Omega_{\alpha \beta} q^{\alpha} q^{\beta}-J^{2}},
$$

where $J$ is the eigenvalue corresponding to the $\mathrm{U}(1)_{L} \subset \mathrm{SU}(2)_{L}$ symmetry in the convention that it is half-integer valued. The microscopic formula (4.88) is only valid in the limit where $n$ is much larger than the other charges. Comparison with (4.86) leads to the identification $Q=n, \tilde{Q}^{\alpha}=q^{\alpha}$ and $J_{\psi}=J$ which explains the normalizations in (4.85).

One can also construct black string solutions with a Taub-NUT base space and asymptotics $\mathbb{R} \times S^{1} \times S^{1} \times \mathbb{R}^{3}$. Although the full solution will be very different from (4.83), the near horizon geometry will be the same. We can then compare (4.86) for $m \neq 1$ with the leading order contribution of the entropy calculated in the microscopic setting corresponding to the Taub-NUT solution. This setting is an F-theory background $\mathbb{R} \times S^{1} \times T N_{m} \times C Y_{3}$, where $T N_{m}$ is a Taub-NUT spacetime with NUT charge $m$. The D3-brane is still wrapped on $S^{1} \times C$ and given $n$ units of momentum along the circle after compactification on $C Y_{3}$. The Taub-NUT breaks the $\mathrm{SO}(4)$ symmetry to $\mathrm{U}(1)_{L} / \mathbb{Z}_{m} \times \mathrm{SU}(2)_{R}$. In [64] the entropy for this setting is calculated in the dual picture that one gets starting from type IIB, T-dualizing along the NUT-circle and then lifting it to M-theory. The M-theory picture is then given by an M5-brane wrapped around $S^{1} \times\left(m B_{2}+\hat{C}\right)$, where $\hat{C}=\pi^{-1}(C)$. The entropy is calculated using the MSW formula [65] and is to leading order equal to

$$
S=2 \pi \sqrt{\frac{1}{2} m n \Omega_{\alpha \beta} q^{\alpha} q^{\beta}-J^{2}},
$$

where $J$ is the eigenvalue corresponding to the $\mathrm{U}(1)_{L} / \mathbb{Z}_{m}$ symmetry along the NUT-circle. The microscopic formula (4.89) is also only valid under certain conditions. Besides the 
Cardy limit in which $n$ has to be much larger than the other charges, we also have that $q^{\alpha} \gg m c^{\alpha}$, where $c^{\alpha}$ comes from the expansion of the first Chern class of the base space: $c_{1}\left(B_{2}\right)=c^{\alpha} \omega_{\alpha}$. The latter condition is needed to make the divisor $m B_{2}+\hat{C}$ very ample. Comparing (4.86) with (4.89) we find that $m$ has to be an integer and that we can identify $Q=n, \tilde{Q}^{\alpha}=q^{\alpha}$ and $J_{\psi}=J$ in the limits where (4.89) is valid. To fully compare this microscopic setting with a macroscopic solution, we of course have to construct the solution with Taub-NUT base space $\mathcal{B}$, but we will leave this for future work [66].

Uplift black ring. A five-dimensional black ring solution [3, 59, 67] is asymptotically flat, has a regular horizon with topology $S^{1} \times S^{2}$ and near horizon geometry $A d S_{3} \times S^{2}$. We will show that the $6 \mathrm{~d}$ uplift has horizon $S^{1} \times S^{3}$ and near horizon geometry $A d S_{3} \times S^{3}$, and is thus consistent with the classification we stated at the beginning of this section. A more general discussion of uplifts of black rings in connection to supertubes was given in [68]. Essentially, our discussion below is a particular and simple case of theirs, so we will be rather brief here and only focus on the near horizon geometry.

To be specific, we take the black ring solution from [67] written in certain coordinates $v, r, \psi^{\prime}, \phi^{\prime}, \theta$ and $\chi$ and in which the near horizon limit is taken by redefining $r=\epsilon L \tilde{r} / R$, $v=\tilde{v} / \epsilon$ (where $L$ and $R$ are certain constants) and sending $\epsilon \rightarrow 0$. In this limit, the metric becomes

$$
d s_{5}^{2}=2 d \tilde{v} d \tilde{r}+\frac{4 L}{q} \tilde{r} d \tilde{v} d \psi^{\prime}+L^{2} d \psi^{\prime 2}+\frac{q^{2}}{4}\left(d \theta^{2}+\sin ^{2}(\theta) d \chi^{2}\right),
$$

where $q$ is another constant. In the same limit, the vector field (in the conventions of section 4.3) is given by

$$
A=\frac{1}{2 q}\left[3 Q-q^{2}\right] \frac{C_{1}}{r} d r-\frac{q}{2} \cos (\theta) d \chi
$$

where $Q, C_{1}$ are other constants and where we have added the exact form

$$
\frac{1}{2 q}\left(3 Q-3 q^{2}\right) d \psi^{\prime}+\frac{q}{2} d \phi^{\prime}+\frac{q}{2} d \psi^{\prime}
$$

to the expression in [67]. From section 4.3 we see that the metric of the six-dimensional uplift of a solution in the timelike class of minimal five-dimensional supergravity is given by

$$
d s_{6}^{2}=(d u+A)^{2}+d s_{5}^{2} .
$$

Redefining $d u=-\frac{q}{2} d u^{\prime}-\frac{1}{2 q}\left[3 Q-q^{2}\right] \frac{C_{1}}{r} d r$ (this can be done in the full solution), the metric becomes

$$
d s_{6}^{2}=\frac{q^{2}}{4}\left(d u^{\prime}+\cos (\theta) d \chi\right)^{2}+2 d \tilde{v} d \tilde{r}+\frac{4 L}{q} \tilde{r} d \tilde{v} d \psi^{\prime}+L^{2} d \psi^{\prime 2}+\frac{q^{2}}{4}\left(d \theta^{2}+\sin ^{2}(\theta) d \chi^{2}\right) .
$$

The $u^{\prime}, \theta$ and $\chi$ part form the round metric on $S^{3}$, where we need $0 \leq u^{\prime}<4 \pi$ to make it regular. The near horizon geometry of the uplift of the black ring is thus $A d S_{3} \times S^{3}$. 
Black lens. In minimal five-dimensional supergravity one also has solutions that have a horizon with lens space topology $L(m, 1)=S^{3} / \mathbb{Z}_{m}$ and are asymptotically flat $[4,6]$. In section 4.3 is described how such solutions can be uplifted to six dimensions. They will fall in the class with a Gibbons-Hawking base space and have harmonic functions of the form (4.70) with $m$ centers. Their near horizon geometry will locally be given by $A d S_{3} \times S^{3} / \mathbb{Z}_{m}$ and their asymptotics will be $\mathbb{R} \times S^{1} \times \mathbb{R}^{4}$. Note that solutions with the same near horizon geometry but different asymptotics are given by the previously described black string with asymptotics $\mathbb{R} \times S^{1} \times \mathbb{R}^{4} / \mathbb{Z}_{m}$ and by a black string with a Taub-NUT space as base space which will have asymptotics $\mathbb{R} \times S^{1} \times S^{1} \times \mathbb{R}^{3}$.

\section{6 pp-waves}

A pp-wave is characterised by the existence of a covariant constant null vector field. This vector field is necessarily a Killing vector field. Requiring the null Killing vector field $\partial_{v}$ of the general solution to be covariantly constant implies that

$$
d\left[H^{-1}(d u+\beta)\right]=0,
$$

which is equivalent to $\mathcal{D} \beta=0$ and $H^{-1}(\mathcal{D} H)=-\dot{\beta}$.

A first class of pp-waves is given by non-twisting solutions of section 4.1 with $H=$ $H(u)$. It follows from the construction of the coordinates in section 3.1 that in this case we may choose $H=1$ by redefining $u$ in (3.2), such that the solution becomes

$$
\begin{aligned}
& d s_{6}^{2}=2 d u\left(d v+\omega+\frac{1}{2} \mathcal{F} d u\right)+d s_{4}^{2}, \\
& \hat{G}^{\alpha}=-e^{+} \wedge d u \wedge \tilde{d} j^{\alpha}+d u \wedge\left[j^{\alpha} \psi-j^{\alpha}(\tilde{d} \omega)^{-}+H_{\mathrm{SD}}^{\alpha}\right]+\star_{4} \tilde{d} j^{\alpha} .
\end{aligned}
$$

The flat base space solution derived in section 4.1 is an example of a pp-wave when we take the functions $P^{\alpha}$ and $Q^{\alpha}$ such that $P_{\alpha} P^{\alpha}=1, P_{\alpha} Q^{\alpha}=0$ and $Q_{\alpha} Q^{\alpha}=0$. This is only possible when $Q^{\alpha}=0$. Even with all these extra conditions, the tensor branch of the theory provides a generalization of the solution in [7] since in general the scalars are still $u$-dependent and the two-forms $H_{\mathrm{SD}}^{\alpha}$ are non-vanishing. To simplify a bit more we choose $\eta=1, W_{1}=0$ and take $C_{3}$ such that $\Omega_{\alpha \beta} C_{3}^{\alpha} P^{\beta}=2$. Transforming to Cartesian coordinates (see [37]) we find that

$$
\omega=\frac{1}{4} \Omega_{\alpha \beta} C_{3}^{\alpha} P^{\beta} r^{2} \sigma_{R}^{3}=x^{1} d x^{2}-x^{2} d x^{1}+x^{3} d x^{4}-x^{4} d x^{3} .
$$

For this solution also $(\tilde{d} \omega)^{-}=0$. Performing now the coordinate transformation

$$
\begin{aligned}
& x^{1}=\sin (u) y^{1}-\cos (u) y^{2}, \\
& x^{2}=\cos (u) y^{1}+\sin (u) y^{2}, \\
& x^{3}=\cos (u) y^{3}+\sin (u) y^{4}, \\
& x^{4}=-\sin (u) y^{3}+\cos (u) y^{4},
\end{aligned}
$$


we obtain the plane wave solution

$$
\begin{aligned}
d s_{6}^{2} & =2 d u d v+\left(\mathcal{F}-\delta_{m n} y^{m} y^{n}\right) d u^{2}+\delta_{m n} d y^{m} d y^{n}, \\
\hat{G}^{\alpha} & =\left(C_{3}^{\alpha}-2 P^{\alpha}\right) d u \wedge\left[d y^{1} \wedge d y^{2}+d y^{3} \wedge d y^{4}\right], \\
j^{\alpha} & =P^{\alpha}(u) .
\end{aligned}
$$

A second class of pp-waves are the solutions in which $\hat{G}^{\alpha}$ vanish. A subset of these solutions is given by the vacuum solutions in which also the (physical) scalars vanish. From (3.13) we find that $\hat{G}^{\alpha}=0$ is equivalent to

$$
\begin{aligned}
H^{-1} \mathcal{D} H & =-\dot{\beta}, \\
\mathcal{D} \beta & =0, \\
\mathcal{D} j^{\alpha} & =0, \\
H \psi & =(\mathcal{D} \omega)^{-}, \\
H_{\mathrm{SD}}^{\alpha} & =0 .
\end{aligned}
$$

The first and second conditions define a pp-wave. The set of equations (4.100) will be hard to solve without extra assumptions. Of course, one can again look at the subclasses of non-twisting and $u$-independent solutions. As an example of a non-twisting solution that falls in this class, we can take (4.99) with $C_{3}^{\alpha}=2 P^{\alpha}$. In this case the solution simplifies to

$$
\begin{aligned}
d s_{6}^{2} & =2 d u d v+\left(C_{5}(u)-\frac{1}{2} C_{4}(u) \frac{1}{r^{2}}+\frac{1}{4} \Omega_{\alpha \beta} \dot{P}^{\alpha} \dot{P}^{\beta} r^{2}\right) d u^{2}+\delta_{m n} d y^{m} d y^{n}, \\
\hat{G}^{\alpha} & =0 \\
j^{\alpha} & =P^{\alpha}(u),
\end{aligned}
$$

where we still have the condition $P_{\alpha} P^{\alpha}=1$.

One last example we consider is not a proper pp-wave, but it is a black string with traveling waves that carry momentum along the string $[69,70]$. This solution falls into the non-twisting class with flat base space (section 4.1). Taking $W_{1}=C_{3}^{\alpha}=C_{5}=0$ and $P^{\alpha}$ and $Q^{\alpha}$ constant such that $\Omega_{\alpha \beta} P^{\alpha} P^{\beta}=1$, we find the solution

$$
\begin{aligned}
d s_{6}^{2} & =2 H^{-1} d u\left(d v-\frac{1}{4} C_{4}(u) \frac{1}{r^{2}} d u\right)+H d s_{4}^{2}, \\
\hat{G}^{\alpha} & =-d v \wedge d u \wedge \tilde{d}\left(H^{-1} j^{\alpha}\right)+\star_{4} \tilde{d}\left(j^{\alpha} H\right), \\
j^{\alpha} H & =P^{\alpha}+\frac{Q^{\alpha}}{r^{2}} .
\end{aligned}
$$

In the limit $r \rightarrow \infty$ this solution is the same as (4.101) with $C_{5}=\dot{P}^{\alpha}=0$, but note that the full solution is very different, mainly because we now have non-vanishing three-forms to support the black string and also the scalars depend on the base space instead of on $u$. For a further discussion of this kind of geometry, see e.g. the original references [69, 70]. The horizon of these solutions become singular however, as discussed e.g. in [71-73]. Perhaps a more interesting class of solutions are the traveling wave deformations of smooth horizonless solutions, such as discussed e.g. in [56]. It could be interesting to extend the analysis of [56] to the present setup where more tensor multiplets are present. 


\section{Attractor mechanism}

In this section we study the attractor mechanism [44, 45] in six-dimensional $(1,0)$ supergravity coupled to tensor multiplets. We first repeat the near horizon analysis $[46,47]$ to show that the scalars near the horizon can be expressed in terms of the charges of the black object. After that we derive a "flow" equation for $u$-independent solutions which in certain simplifying cases explains this attractor mechanism from the full geometry perspective. Our version of the attractor flow is consistent with the five-dimensional flow equation in [74]. A general proof of the attractor mechanism for single, charged, static, flat $p$-brane solutions in $d$ dimensions is given in [48]. Some of the solutions we consider will also be of this type, but not all of them.

\subsection{Near horizon analysis}

We consider the near horizon geometries of black objects which are locally $A d S_{3} \times S^{3}$ (so we consider one of the three possible cases). In [8] it is shown that in this geometry the tensor multiplet scalars $j^{\alpha}$ are constants and the tensors $H^{M}$ of the tensor multiplets vanish. Integrating over the spherical part of the solution (e.g. in (4.83) this part is parametrized by $\psi, \phi$ and $\theta$ ) implies that the charges that correspond to $\hat{G}^{\alpha}=j^{\alpha} H$ are equal to

$$
\tilde{Q}^{\alpha}=j^{\alpha} k .
$$

Using $j_{\alpha} j^{\alpha}=1$, we find that

$$
k=\sqrt{\Omega_{\alpha \beta} \tilde{Q}^{\alpha} \tilde{Q}^{\beta}}
$$

and

$$
j^{\alpha}=\frac{\tilde{Q}^{\alpha}}{\sqrt{\Omega_{\beta \gamma} \tilde{Q}^{\beta} \tilde{Q}^{\gamma}}} .
$$

Hence in the near horizon geometry the scalars take a value completely expressed in terms of the charges related to the three-forms.

\subsection{Flow equation}

It would be nice to be able to see the scalar values arise from the flow of a central charge as one usual can (e.g. [74]). We will derive this "flow" equation for $u$-independent solutions. The general flow is complicated, but we consider a class of solutions where it simplifies. To derive the flow equation, we need two ingredients: the Bianchi identity and supersymmetry. The part of the three-forms with three legs on the base space is generally what corresponds to the charges, hence we will derive an equation for the scalars and $\hat{G}_{i j k}^{\alpha}$ (note however, that in the near horizon geometry of the uplift of the black ring, the three-sphere is given by the $u$-circle fibered over an $S^{2}$ in the base space). Using supersymmetry and $u$-independence (4.28), but not specifying the part $\hat{G}_{i j k}^{\alpha}$ we can write

$$
\begin{aligned}
\hat{G}^{\alpha}= & -H e^{+} \wedge e^{-} \wedge \tilde{d}\left(H^{-1} j^{\alpha}\right)+j^{\alpha} H^{-1} e^{+} \wedge \tilde{d} \beta+e^{-} \wedge\left[-j^{\alpha}(\tilde{d} \omega)^{-}+H_{\mathrm{SD}}^{\alpha}\right] \\
& +\frac{1}{6} \hat{G}_{i j k}^{\alpha} \tilde{e}^{i} \wedge \tilde{e}^{j} \wedge \tilde{e}^{k} .
\end{aligned}
$$


We now first consider the Bianchi identity and after that use the tensorini equation to finish the derivation of the flow equation. Since we are only interested in $\hat{G}_{i j k}^{\alpha}$ we will, after applying the exterior derivative on (5.4), only consider the part with four legs on $\mathcal{B}$ :

$$
d \hat{G}^{\alpha} \rightarrow j^{\alpha} H^{-1} d e^{+} \wedge \tilde{d} \beta+d e^{-} \wedge\left[-j^{\alpha}(\tilde{d} \omega)^{-}+H_{\mathrm{SD}}^{\alpha}\right]+d\left(\frac{1}{6} \hat{G}_{i j k}^{\alpha} \tilde{e}^{i} \wedge \tilde{e}^{j} \wedge \tilde{e}^{k}\right) .
$$

Calculating the last term in (5.5) yields

$$
d\left(\hat{G}_{i j k}^{\alpha} \tilde{e}^{i} \wedge \tilde{e}^{j} \wedge \tilde{e}^{k}\right)=\tilde{\nabla}_{l}\left(\hat{G}_{i j k}^{\alpha}\right) \tilde{e}^{l} \wedge \tilde{e}^{i} \wedge \tilde{e}^{j} \wedge \tilde{e}^{k} .
$$

Using that

$$
d e^{-} \rightarrow H^{-1} \tilde{d} \beta, \quad d e^{+} \rightarrow \tilde{d} \omega+\frac{1}{2} \mathcal{F} \tilde{d} \beta,
$$

we can finish the calculation of (5.5):

$$
\begin{aligned}
d \hat{G}^{\alpha} \rightarrow & j^{\alpha} H^{-1}\left(\tilde{d} \omega+\frac{1}{2} \mathcal{F} \tilde{d} \beta\right) \wedge \tilde{d} \beta+H^{-1} \tilde{d} \beta \wedge\left[-j^{\alpha}(\tilde{d} \omega)^{-}+H_{\mathrm{SD}}^{\alpha}\right] \\
& +d\left(\frac{1}{6} \hat{G}_{i j k}^{\alpha} \tilde{e}^{i} \wedge \tilde{e}^{j} \wedge \tilde{e}^{k}\right) \\
= & H^{-1}\left[j^{\alpha}(\tilde{d} \omega)^{+}+\frac{1}{2} j^{\alpha} \mathcal{F} \tilde{d} \beta+H_{\mathrm{SD}}^{\alpha}\right] \wedge \tilde{d} \beta+d\left(\frac{1}{6} \hat{G}_{i j k}^{\alpha} \tilde{e}^{i} \wedge \tilde{e}^{j} \wedge \tilde{e}^{k}\right) .
\end{aligned}
$$

With (5.6) this can be rewritten as

$$
d \hat{G}^{\alpha} \rightarrow\left[\frac{1}{4}\left(\mathcal{G}^{+\alpha}\right)_{l i}(\tilde{d} \beta)_{j k}+\frac{1}{6} \tilde{\nabla}_{l}\left(\hat{G}_{i j k}^{\alpha}\right)\right] \tilde{e}^{l} \wedge \tilde{e}^{i} \wedge \tilde{e}^{j} \wedge \tilde{e}^{k} .
$$

The Bianchi identity implies that

$$
\frac{1}{6} \tilde{\nabla}_{l}\left(\hat{G}_{i j k}^{\alpha}\right) \epsilon^{l i j k}=-\frac{1}{4}\left(\mathcal{G}^{+\alpha}\right)_{l i}(\tilde{d} \beta)_{j k} \epsilon^{l i j k} .
$$

From supersymmetry (4.28) it follows that

$$
\hat{G}_{i j k}^{\alpha}=\tilde{d}\left(j^{\alpha} H\right)_{l} \epsilon^{l}{ }_{i j k}
$$

such that

$$
\partial_{l}\left(j^{\alpha}\right) \Omega_{\alpha \beta} \hat{G}_{i j k}^{\beta} \epsilon^{l i j k}=-6 H g_{\alpha \beta} \partial_{l}\left(j^{\alpha}\right) \partial^{l}\left(j^{\beta}\right) .
$$

Combining this with (5.10) results in

$$
\frac{1}{6} \tilde{\nabla}_{l}\left(\Omega_{\alpha \beta} j^{\alpha} \hat{G}_{i j k}^{\beta}\right) \epsilon^{l i j k}=-H g_{\alpha \beta} \partial_{l}\left(j^{\alpha}\right) \partial^{l}\left(j^{\beta}\right)-j_{\alpha} \mathcal{G}^{+\alpha} \cdot \tilde{d} \beta .
$$

This is the flow equation for $u$-independent solutions. In principle one can also do this derivation for the most general solution, but the resulting equation does not put a strong contraint on a flow. Even for the most general $u$-independent solution the meaning of (5.13) is not very clear. However, when also either $\tilde{d} \beta$ or $j_{\alpha} \mathcal{G}^{+\alpha}$ vanish, the right-hand side of (5.13) is non-positive since $g_{\alpha \beta}$ is positive definite. This implies a monotonicity 
property of the quantity $\frac{1}{6} \Omega_{\alpha \beta} j^{\alpha} \hat{G}_{i j k}^{\beta} \epsilon^{l i j k}$. When one derives the flow equation for the most general solution there might be other special cases in which the equation implies a monotonicity property of a quantity. One can also derive an equation for other components of $\hat{G}^{\alpha}$ and examine what this equation would imply for black ring solutions. As a check, we show in appendix $\mathrm{E}$ that when one performs a Kaluza-Klein reduction along the $u$-circle (section 4.3), the flow equation (5.13) reduces to the five-dimensional flow equation derived in [74].

If we let $V \subset \mathcal{B}$ we can define the charges

$$
\tilde{Q}^{\alpha}=\frac{1}{12 \sqrt{2} \pi^{2}} \int_{\partial V} d S \hat{G}_{i j k}^{\alpha} n_{l} \epsilon^{l i j k}
$$

where $n$ is a unit vector perpendicular to $\partial V$ and pointing outward. The quantity (5.14) is for the black string solution in (4.83) equal to the charge defined in (4.85). We can also introduce the central charge

$$
Z(V) \equiv \frac{1}{12 \sqrt{2} \pi^{2}} \int_{\partial V} d S \Omega_{\alpha \beta} j^{\alpha} \hat{G}_{i j k}^{\beta} n_{l} \epsilon^{l i j k},
$$

which, in case the scalars are independent of the region $\partial V$, reduces to

$$
Z(V)=j_{\alpha} \tilde{Q}^{\alpha}
$$

This is the central charge that follows from the supersymmetry algebra [75]. When we have regions $V_{2} \subset V_{1}$, we can use (5.13) to show that

$$
Z\left(V_{1}\right)-Z\left(V_{2}\right)=\frac{1}{2 \sqrt{2} \pi^{2}} \int d^{4} x \sqrt{h}\left(-H g_{\alpha \beta} \partial_{l}\left(j^{\alpha}\right) \partial^{l}\left(j^{\beta}\right)-\frac{1}{2} j_{\alpha} \mathcal{G}^{+\alpha} \cdot \tilde{d} \beta\right) .
$$

In case either $j_{\alpha} \mathcal{G}^{+\alpha}$ or $\tilde{d} \beta$ vanishes, the central charge is monotonically decreasing as we move outwards. If this is not the case, the flow equation does not provide a strong constraint on the flow.

\subsection{One-centered Gibbons-Hawking}

When we take a general solution of section 4.4 with harmonic functions of the form (4.70) and one center, we actually have a clear radial direction such that a natural choice of subspaces $V \subset \mathcal{B}$ is $r \leq r_{0}$. For the general case

$$
Z\left(r_{0}\right)=\frac{1}{12 \sqrt{2} \pi^{2}} \int_{\partial V} d S \Omega_{\alpha \beta} j^{\alpha} \hat{G}_{i j k}^{\beta} n_{l} \epsilon^{l i j k}=\Omega_{\alpha \beta} j^{\alpha} \tilde{Q}^{\beta}
$$

and from (5.17) we find that when either $j_{\alpha} \mathcal{G}^{+\alpha}$ or $\tilde{d} \beta$ vanishes

$$
\begin{aligned}
\partial_{r} Z(r) & =\frac{1}{2 \sqrt{2} \pi^{2}} \partial_{r} \int d^{4} x \sqrt{h}\left[-H g_{\alpha \beta} \partial_{l}\left(j^{\alpha}\right) \partial^{l}\left(j^{\beta}\right)\right] \\
& =-4 \sqrt{2} r^{2} H_{2} H g_{\alpha \beta} \partial_{r}\left(j^{\alpha}\right) \partial^{r}\left(j^{\beta}\right),
\end{aligned}
$$


which is non-positive. Notice that we can write this as

$$
r \partial_{r} Z=-4 \sqrt{2} r^{3} H_{2} H^{2} \epsilon,
$$

where

$$
\epsilon=\hat{g}^{r r} g_{\alpha \beta} \partial_{r}\left(j^{\alpha}\right) \partial_{r}\left(j^{\beta}\right)
$$

is the energy density of the scalar fields. Note that near the horizon $H_{2} \sim \frac{1}{r^{2}}$ which implies that the proper distance to the horizon blows up. Together with a finite area of the horizon, this implies that $\epsilon=0$ because otherwise the energy of the scalar fields would diverge. Hence from (5.20) we find that at the horizon we get

$$
r \partial_{r} Z=0
$$

which is the spacetime form of the attractor formula.

For the most general solution with harmonic functions of the form (4.70), the charge (5.14) reduces in the near horizon limit $r \rightarrow 0$ to

$$
\tilde{Q}^{\alpha} \rightarrow 4 \sqrt{2}\left(\mu^{\alpha}-\frac{1}{m} q p^{\alpha}\right) .
$$

In the same limit

$$
j^{\alpha} \rightarrow \frac{\mu^{\alpha}-\frac{1}{m} q p^{\alpha}}{\sqrt{\Omega_{\beta \gamma}\left(\mu^{\beta}-\frac{1}{m} q p^{\beta}\right)\left(\mu^{\gamma}-\frac{1}{m} q p^{\gamma}\right)}}=\frac{\tilde{Q}^{\alpha}}{\sqrt{\Omega_{\beta \gamma} \tilde{Q}^{\beta} \tilde{Q}^{\gamma}}}
$$

which is indeed the value we found in (5.3). This is also true for the cases where the flow is more complicated.

\section{Outlook}

We derived and analyzed the general local form of supersymmetric solutions of $(1,0)$ supergravity coupled to tensor multiplets, and studied examples of BPS black strings and pp-waves with non-trivial scalar profiles. There are many interesting extensions and generalizations, such as the study of bound states of black strings, and the construction of new microstate geometries and their dual CFT states. It would also be interesting to repeat the general analysis to the case with hypermultiplets and vector multiplets.

We solved the equations of motion completely in certain simplifying cases and studied the attractor flow for $u$-independent solutions. Something that might be interesting as well is to see if there are also attractor mechanisms for the hyperscalars. For maximally supersymmetric solutions this is certainly the case [8], but it is not so clear when the solutions have less Killing spinors.

While we have studied to some extent the embedding in type IIB and in F-theory, it would be nice to study better the microscopic analysis of the black string solutions in F-theory. In particular, the near horizon geometry of black strings leads to new dual $(0,4)$ CFTs that are yet to be constructed and analyzed. For the case of minimal supergravity, corresponding to F-theory compactified on a $\mathrm{CY}_{3}$ with base space $\mathbb{P}^{2}$, this was done in [22], see also [21] for earlier work, and [23-25] for more recent work. 


\section{Acknowledgments}

We would like to thank Kilian Mayer and Thomas Grimm for useful discussions. We are also grateful to Kilian Mayer for reading and commenting this document. We also thank many of the participants of the "Strings, Geometry and Black Holes" conference (Eurostrings 2018) in London, for useful comments and feedback on the poster presentation of this paper. This work was supported in part by the D-ITP consortium, a program of the Netherlands Organization for Scientific Research (NWO) that is funded by the Dutch Ministry of Education, Culture and Science (OCW), and by the NWO Graduate Programme.

\section{A Integrability conditions}

In this appendix we derive which equations of motion are implied by the integrability conditions of the theory. Denote the scalar equations of motion by $(E j)^{M}=0$, the threeform equations of motion by $(E \hat{G})_{\alpha}^{\mu \nu}=\hat{\nabla}_{\lambda}\left(g_{\alpha \beta} \hat{G}^{\beta} \lambda \mu \nu\right)=0$ and the Einstein equation by $E_{\mu \nu}=0$. Contracting the integrability of the gravitino Killing spinor equation with $\gamma^{\nu}$ yields $^{5}$

$$
\gamma^{\nu}\left[\mathcal{D}_{\mu}, \mathcal{D}_{\nu}\right] \epsilon=E_{\mu \nu} \gamma^{\nu} \epsilon+\frac{1}{8} j^{\alpha}(E \hat{G})_{\alpha \rho \sigma} \hat{g}_{\mu \nu} \gamma^{\nu \rho \sigma} \epsilon-\frac{1}{4} j^{\alpha}(E \hat{G})_{\alpha \mu \nu} \gamma^{\nu} \epsilon=0
$$

Assuming the three-form equations of motion it follows that

$$
E_{\mu \nu} \gamma^{\nu} \epsilon=0
$$

In the null-basis $\gamma_{+} \epsilon=0$, thus we observe that (A.2) implies that

$$
E_{\mu+}=E_{\mu 1}=E_{\mu 2}=E_{\mu 3}=E_{\mu 4}=0 .
$$

Hence, only the $E_{--}$component is not determined by the integrability conditions.

The integrability of the tensorini Killing spinor equation contracted with $\gamma^{\mu}$ and expressed in the equations of motion yields ${ }^{6}$

$$
\gamma^{\mu}\left[\mathcal{D}_{\mu}, T_{\nu}^{M} \gamma^{\nu}-\frac{1}{12} H_{\nu \rho \sigma}^{M} \gamma^{\nu \rho \sigma}\right] \epsilon=(E j)^{M} \epsilon+\frac{1}{2} x_{\alpha}^{M}(E \hat{G})_{\mu \nu}^{\alpha} \gamma^{\mu \nu} \epsilon=0
$$

Assuming the three-form equations of motion, it follows that (A.4) is equivalent to the scalar equations of motion.

\footnotetext{
${ }^{5}$ This condition is derived in the PhD thesis of Mehmet Akyol (Kings college).

${ }^{6}$ This condition is derived in the PhD thesis of Mehmet Akyol (Kings college), although there the wrong scalar equation of motion is used.
} 


\section{B Spin connection}

In this section, the components $i, j, \ldots$ will refer to the part of the six-dimensional vielbein $\hat{e}^{i}$, unless they are components of base space objects $\tilde{\omega}$ and $\tilde{e}^{i}$. Using metric compatibility, so anti-symmetry of the connection

$$
\begin{aligned}
\hat{\omega}_{i j} & =-\hat{\omega}_{j i}, \\
\hat{\omega}_{+i} & =-\hat{\omega}_{i+}, \\
\hat{\omega}_{-i} & =-\hat{\omega}_{i-}, \\
\hat{\omega}_{++} & =0, \\
\hat{\omega}_{--} & =0, \\
\hat{\omega}_{+-} & =-\hat{\omega}_{-+},
\end{aligned}
$$

and vanishing torsion, a straightforward calculation yields that the spin connection is given by

$$
\begin{aligned}
\hat{\omega}^{+}{ }_{i}= & \frac{1}{2}\left(\mathcal{D} \omega+\frac{1}{2} \mathcal{F} \mathcal{D} \beta\right)_{i j} \hat{e}^{j}-\frac{1}{2} \partial_{u}\left(H h_{m n}\right) \tilde{e}_{i}^{m} \tilde{e}_{j}^{n} \hat{e}^{j} \\
& -H\left(\dot{\omega}+\frac{1}{2} \mathcal{F} \dot{\beta}-\frac{1}{2} \mathcal{D} \mathcal{F}\right)_{i} e^{-}-\frac{1}{2}\left(H^{-1} \mathcal{D} H+\dot{\beta}\right)_{i} e^{+}, \\
\hat{\omega}^{-}{ }_{i}= & \frac{1}{2} H^{-1}(\mathcal{D} \beta)_{i j} \hat{e}^{j}-\frac{1}{2}\left(H^{-1} \mathcal{D} H+\dot{\beta}\right)_{i} e^{-}, \\
\hat{\omega}^{+}{ }_{+}= & -\frac{1}{2}\left(H^{-1} \mathcal{D} H+\dot{\beta}\right)_{i} \hat{e}^{i}, \\
\hat{\omega}^{-}= & \frac{1}{2}\left(H^{-1} \mathcal{D} H+\dot{\beta}\right)_{i} \hat{e}^{i}, \\
\hat{\omega}^{i}{ }_{j}= & \tilde{\omega}^{i}{ }_{j}+\frac{1}{2} H^{-1}(\mathcal{D} H)_{j} \hat{e}^{i}-\frac{1}{2} H^{-1}(\mathcal{D} H)^{i} \delta_{j k} \hat{e}^{k}+\frac{1}{2} H^{1 / 2}\left(\beta \wedge \dot{\tilde{e}}^{i}\right)_{k j} \hat{e}^{k}+\frac{1}{2} H^{1 / 2}\left(\beta \wedge \dot{\tilde{e}}_{k}\right)_{j}{ }_{j} \hat{e}^{k} \\
& +\frac{1}{2} H^{1 / 2}\left(\beta \wedge \dot{\tilde{e}}_{j}\right)_{k}{ }_{k} \hat{e}^{k}-\frac{1}{2} H^{-1}(\mathcal{D} \beta)^{i}{ }_{j} e^{+}-\frac{1}{2}\left(\mathcal{D} \omega+\frac{1}{2} \mathcal{F} \mathcal{D} \beta\right)^{i} e^{-}-H\left(\partial_{u} \tilde{e}_{m}^{i}\right)^{i} \tilde{e}_{j]}^{m} e^{-} .
\end{aligned}
$$

\section{C $\quad R_{--}$component of the Ricci tensor}

In this section, the components $i, j, \ldots$ will at first refer to the part of the six-dimensional vielbein $\hat{e}^{i}$, unless they are components of base space objects $\tilde{\omega}$ and $\tilde{e}^{i}$ or indicated as $\tilde{i}$. We would like to calculate the -- component of the Ricci tensor:

$$
R_{--}=R_{-+-}^{+}+R_{-i-}^{i}
$$

We calculate the curvature two-form via the spin-connection:

$$
\begin{aligned}
R^{i}{ }_{-} & =d \hat{\omega}^{i}{ }_{-}+\hat{\omega}^{i}{ }_{j} \wedge \hat{\omega}^{j}{ }_{-}+\hat{\omega}^{i}{ }_{-} \wedge \hat{\omega}^{-}{ }_{-}, \\
R^{+}{ }_{-} & =d \hat{\omega}^{+}{ }_{-}+\hat{\omega}^{+}{ }_{i} \wedge \hat{\omega}^{i}{ }_{-},
\end{aligned}
$$


where we will only keep the $R_{-j-}^{i}$ and $R^{+}{ }_{-+-}$components. A straightforward (but lengthy) calculation yields

$$
\begin{aligned}
R_{-}^{+}= & 0, \\
R_{-}^{i} \rightarrow & \left\{H \partial_{u}\left[\frac{1}{2}\left(\mathcal{D} \omega+\frac{1}{2} \mathcal{F} \mathcal{D} \beta\right)_{j}^{i}-\frac{1}{2} \partial_{u}\left(H h_{m n}\right) \tilde{e}^{m i} \tilde{e}_{j}^{n}\right]\right. \\
& +\left[\frac{1}{2}\left(\mathcal{D} \omega+\frac{1}{2} \mathcal{F} \mathcal{D} \beta\right)_{k}^{i}-\frac{1}{2} \partial_{u}\left(H h_{m n}\right) \tilde{e}^{m i} \tilde{e}_{k}^{n}\right]\left[\frac{1}{2} \dot{H} \delta_{j}^{k}+H\left(\partial_{u} \tilde{e}_{o}^{k}\right) \tilde{e}_{j}^{o}\right] \\
& +\frac{1}{2} H^{-1}\left(\partial_{\tilde{j}} H\right)\left(\dot{\omega}+\frac{1}{2} \mathcal{F} \dot{\beta}-\frac{1}{2} \mathcal{D} \mathcal{F}\right)^{\tilde{i}}+\tilde{\nabla} \tilde{j}_{j}\left[\left(\dot{\omega}+\frac{1}{2} \mathcal{F} \dot{\beta}-\frac{1}{2} \mathcal{D} \mathcal{F}\right)^{i}\right] \\
& -\partial_{u}\left[H\left(\dot{\omega}+\frac{1}{2} \mathcal{F} \dot{\beta}-\frac{1}{2} \mathcal{D} \mathcal{F}\right)^{i}\right] \beta_{j}-\frac{3}{2} H\left(\dot{\omega}+\frac{1}{2} \mathcal{F} \dot{\beta}-\frac{1}{2} \mathcal{D} \mathcal{F}\right)^{i}\left(H^{-1} \mathcal{D} H+\dot{\beta}\right)_{j} \\
& -\frac{1}{2} H\left(H^{-1} \mathcal{D} H+\dot{\beta}\right)^{i}\left(\dot{\omega}+\frac{1}{2} \mathcal{F} \dot{\beta}-\frac{1}{2} \mathcal{D} \mathcal{F}\right)_{j} \\
& +H\left(\dot{\omega}+\frac{1}{2} \mathcal{F} \dot{\beta}-\frac{1}{2} \mathcal{D} \mathcal{F}\right)^{k}\left[\frac{1}{2} H^{-1}(\mathcal{D} H)_{k} \delta_{j}^{i}-\frac{1}{2} H^{-1}(\mathcal{D} H)^{i} \delta_{k j}+\frac{1}{2} H^{1 / 2}\left(\beta \wedge \dot{\tilde{e}}^{i}\right)_{j k}\right. \\
& \left.+\frac{1}{2} H^{1 / 2}\left(\beta \wedge \dot{\tilde{e}}_{j}\right)_{k}^{i}+\frac{1}{2} H^{1 / 2}\left(\beta \wedge \dot{\tilde{e}}_{k}\right)_{j}^{i}\right]-\left[\frac{1}{2}\left(\mathcal{D} \omega+\frac{1}{2} \mathcal{F} \mathcal{D} \beta\right)_{k}^{i}+H\left(\partial_{u} e_{m}^{[i} e_{k]}^{m}\right]\right. \\
& \left.\times\left[\frac{1}{2}\left(\mathcal{D} \omega+\frac{1}{2} \mathcal{F} \mathcal{D} \beta\right)^{k}-\frac{1}{2} \partial_{u}\left(H h_{m n}\right) \tilde{e}^{m k} \tilde{e}_{j}^{n}\right]\right\} \hat{e}^{j} \wedge e^{-} .
\end{aligned}
$$

Taking the $R_{-i-}^{i}$ components, summing over $i$ and rewriting everything in components with respect to the vielbein $\tilde{e}^{i}$ yields

$$
\begin{aligned}
R_{--}= & \star_{4} \mathcal{D} \star_{4}\left(\dot{\omega}+\frac{1}{2} \mathcal{F} \dot{\beta}-\frac{1}{2} \mathcal{D} \mathcal{F}\right)-2\left(\dot{\omega}+\frac{1}{2} \mathcal{F} \dot{\beta}-\frac{1}{2} \mathcal{D} \mathcal{F}\right)^{m} \partial_{u}\left(\beta_{m}\right) \\
& +\frac{1}{4} H^{-2}\left(\mathcal{D} \omega+\frac{1}{2} \mathcal{F} \mathcal{D} \beta\right)_{i k}\left(\mathcal{D} \omega+\frac{1}{2} \mathcal{F} \mathcal{D} \beta\right)^{i k} \\
& -\frac{1}{2} H h^{m n} \partial_{u}^{2}\left(H h_{m n}\right)-\frac{1}{4} \partial_{u}\left(H h_{m n}\right) \partial_{u}\left(H h^{m n}\right) .
\end{aligned}
$$

\section{Single string solution}

Taking a single string at the origin, we still have to determine the one-forms $\vec{\chi}, \vec{\beta}$ and $\vec{\omega}$. Solving the equations for $\vec{\chi}, \vec{\beta}$ and $\vec{\omega}$, and assuming the Bubble equations, we find that

$$
\begin{aligned}
\chi_{a} d x^{a} & =m \cos (\theta) d \phi, \\
\beta_{a} d x^{a} & =-q \cos (\theta) d \phi, \\
\omega_{a} d x^{a} & =0 .
\end{aligned}
$$




\section{D.1 Asymptotics}

Taking a single string, we require the metric to asymptote to $\mathbb{R} \times S_{u}^{1} \times \mathbb{R}^{4} / \mathbb{Z}_{m}$. This implies that $m_{\infty}=0$, the functions $H, \mathcal{F} \rightarrow 1$ and the one-forms $\omega, \beta \rightarrow 0$. The limit

$$
\lim _{r \rightarrow \infty} \mathcal{F}=-n_{\infty}+\frac{r}{m} \Omega_{\alpha \beta} p_{\infty}^{\alpha} p_{\infty}^{\beta}+2 \frac{1}{m} \Omega_{\alpha \beta} p_{\infty}^{\alpha} p^{\beta}=1
$$

implies that $p_{\infty}^{\alpha}=0$ and $n_{\infty}=-1$. The limit of the one-form $\beta \rightarrow 0$ implies that $q_{\infty}=0$ such that $\beta \rightarrow \frac{q}{m} d \psi$. This can be absorbed by the coordinate redefinition $d u \rightarrow d u-\frac{q}{m} d \psi$. For the single string, $u$ is periodic, hence we need that

$$
\frac{4 \pi q}{l m} \in \mathbb{Z}
$$

where $l$ is the length of the circle, for this to be well-defined. The limit

$$
\lim _{r \rightarrow \infty} H=\sqrt{\Omega_{\alpha \beta} \mu_{\infty}^{\alpha} \mu_{\infty}^{\alpha}}=1
$$

then implies that

$$
\Omega_{\alpha \beta} \mu_{\infty}^{\alpha} \mu_{\infty}^{\beta}=1
$$

Lastly, the limit of the one-form $\omega \rightarrow 0$ implies that

$$
\lim _{r \rightarrow 0} \omega_{0}=j_{\infty}+\frac{1}{m} \Omega_{\alpha \beta} \mu_{\infty}^{\alpha} p^{\beta}-\frac{1}{2} \frac{q}{m}=0
$$

such that

$$
j_{\infty}=\frac{1}{2} \frac{q}{m}-\frac{1}{m} \Omega_{\alpha \beta} \mu_{\infty}^{\alpha} p^{\beta} .
$$

Hence, to get the correct asymptotics, we need

$$
\Gamma_{\infty}=\left(\mu_{\infty}^{\alpha}, 0,0,0,-1, \frac{1}{2} \frac{q}{m}-\frac{1}{m} \Omega_{\alpha \beta} \mu_{\infty}^{\alpha} p^{\beta}\right)
$$

subject to (D.5). With these values for $\Gamma_{\infty}$, the Bubble equations are automatically satisfied.

\section{E Reduction of the flow equation to five dimensions}

We show that when compactifying along the $u$-circle (as done in section 4.3), the flow equation (5.13) reduces to the flow equation derived in [74], which in our conventions is given $\mathrm{by}^{7}$

$$
\tilde{\nabla}^{l}\left(f^{-1} \mathcal{G}_{I J} X^{I} E_{l}^{J}\right)=f^{-1} \mathcal{G}_{I J} \partial_{l} X^{I} \partial^{l} X^{J}-\frac{1}{4} C_{I J K} X^{I} \Theta^{J} \cdot \Theta^{K},
$$

where

$$
\mathcal{G}_{I J}=\left.\left[-\frac{1}{2} \partial_{X^{I}} \partial_{X^{J}} \log \mathcal{N}\right]\right|_{\mathcal{N}=1}=\frac{9}{2} X_{I} X_{J}-\frac{1}{2} C_{I J K} X^{K}
$$

\footnotetext{
${ }^{7}$ In their conventions $X_{I} \equiv \frac{1}{2} C_{I J K} X^{J} X^{K}$ and $\alpha \cdot \beta=\alpha_{m n} \beta^{m n}$.
} 
and $E_{l}^{I}=F_{l v}^{I}=f^{-1} \partial_{l}\left(f X^{I}\right)$. Some useful identities that follow from $X_{I} X^{I}=1$ are:

$$
\begin{aligned}
\mathcal{G}_{I J} X^{J} & =\frac{3}{2} X_{I}, \\
\partial_{l} X_{I} & =-\frac{2}{3} \mathcal{G}_{I J} \partial_{l} X^{J} .
\end{aligned}
$$

From the cubic potential (4.40) one finds

$$
\begin{aligned}
\mathcal{G}_{\alpha \beta} & =r^{-4 / 3} g_{\alpha \beta}, \\
\mathcal{G}_{0 \beta} & =0, \\
\mathcal{G}_{00} & =\frac{1}{2} r^{8 / 3} .
\end{aligned}
$$

Let's now reduce the terms in the flow equation (5.13) one by one. We start with the left-hand side:

$$
\frac{1}{6} \tilde{\nabla}_{l}\left(\Omega_{\alpha \beta} j^{\alpha} \hat{G}_{i j k}^{\beta}\right) \epsilon^{l i j k} .
$$

Note that the base space in the five- and six-dimensional space has the same metric $d s_{4}^{2}$, so the covariant derivative does not change. The vierbein $\hat{e}^{i}$ is related to a vierbein $e^{i}$ of the $5 \mathrm{~d}$ spatial part via $\hat{e}^{i}=r^{-1 / 3} e^{i}$ and $e^{i}=f^{-1 / 2} \tilde{e}^{i}$. Applying the self-duality condition (2.27) to the ansatz for the three-forms (4.36) relates $G^{\alpha}$ to the two-forms $F^{\alpha}$. In particular when we express

$$
G^{\alpha}=\frac{1}{2} G_{i j}^{\alpha} \tilde{e}^{i} \wedge \tilde{e}^{j} \wedge(d v+\omega)+\frac{1}{6} G_{i j k}^{\alpha} \tilde{e}^{i} \wedge \tilde{e}^{j} \wedge \tilde{e}^{k},
$$

we find that

$$
G_{i j k}^{\alpha}=-f^{-1} r^{-4 / 3} g^{\alpha \beta} \Omega_{\beta \gamma} E_{l}^{\gamma} \epsilon_{i j k}^{l} .
$$

Hence

$$
\hat{G}_{i j k}^{\alpha}=G_{i j k}^{\alpha}=-f^{-1} r^{-4 / 3}\left(2 j^{\alpha} j_{\gamma}-\delta_{\gamma}^{\alpha}\right) E_{l}^{\gamma} \epsilon_{i j k}^{l} .
$$

We then derive that

$$
\frac{1}{6} \tilde{\nabla}_{l}\left(\Omega_{\alpha \beta} j^{\alpha} \hat{G}_{i j k}^{\beta}\right) \epsilon^{l i j k}=-\tilde{\nabla}^{l}\left(f^{-1} r^{-4 / 3} \Omega_{\alpha \beta} j^{\alpha} E_{l}^{\beta}\right) .
$$

Inserting the ansatz for the scalars (4.37) and applying the product rule gives

$$
\frac{1}{6} \tilde{\nabla}_{l}\left(\Omega_{\alpha \beta} j^{\alpha} \hat{G}_{i j k}^{\beta}\right) \epsilon^{l i j k}=-r^{-2 / 3} \tilde{\nabla}^{l}\left(f^{-1} r^{-4 / 3} \Omega_{\alpha \beta} X^{\alpha} E_{l}^{\beta}\right)-f^{-1} r^{-4 / 3} \Omega_{\alpha \beta} X^{\alpha} E_{l}^{\beta} \partial^{l}\left(r^{-2 / 3}\right) .
$$

Using (E.4) we then calculate that

$$
\tilde{\nabla}^{l}\left(f^{-1} \mathcal{G}_{I J} X^{I} E_{l}^{J}\right)=\frac{1}{2} \tilde{\nabla}^{l}\left(f^{-1} r^{4 / 3} E_{l}^{0}\right)+\tilde{\nabla}^{l}\left(f^{-1} r^{-4 / 3} \Omega_{\alpha \beta} X^{\alpha} E_{l}^{\beta}\right)
$$

and combining (E.4) with the definition of $E_{l}^{\beta}$ and $\partial_{l} r=-\frac{3}{4} r^{7 / 3} \partial_{l} X^{0}$, the second term of the right-hand side in (E.10) can be calculated:

$$
f^{-1} r^{-4 / 3} \Omega_{\alpha \beta} X^{\alpha} E_{l}^{\beta} \partial^{l}\left(r^{-2 / 3}\right)=-\frac{1}{2} r^{-2 / 3} f^{-1} \mathcal{G}_{00} \partial_{l}\left(X^{0}\right) \partial^{l}\left(X^{0}\right)-\frac{2}{3} f^{-2} r^{-5 / 3} \partial_{l}(f) \partial^{l}(r) .
$$


Substitution of (E.11) and (E.12) in (E.10) yields

$$
\begin{aligned}
\frac{1}{6} \tilde{\nabla}_{l}\left(\Omega_{\alpha \beta} j^{\alpha} \hat{G}_{i j k}^{\beta}\right) \epsilon^{l i j k}= & -r^{-2 / 3} \tilde{\nabla}^{l}\left(f^{-1} \mathcal{G}_{I J} X^{I} E_{l}^{J}\right)+\frac{1}{2} r^{-2 / 3} \tilde{\nabla}^{l}\left(f^{-1} r^{4 / 3} E_{l}^{0}\right) \\
& +\frac{1}{2} r^{-2 / 3} f^{-1} \mathcal{G}_{00} \partial_{l}\left(X^{0}\right) \partial^{l}\left(X^{0}\right)+\frac{2}{3} f^{-2} r^{-5 / 3} \partial_{l}(f) \partial^{l}(r) .
\end{aligned}
$$

Let us then reduce the first term of the right-hand side of the flow equation (5.13). Inserting the ansatz for the scalars (4.37), applying the product rule and using (E.4) yields

$$
\begin{aligned}
H g_{\alpha \beta} \partial_{l}\left(j^{\alpha}\right) \partial^{l}\left(j^{\beta}\right)= & H \mathcal{G}_{\alpha \beta} \partial_{l}\left(X^{\alpha}\right) \partial^{l}\left(X^{\beta}\right)+2 H r^{2 / 3} \mathcal{G}_{\alpha \beta} X^{\beta} \partial_{l}\left(X^{\alpha}\right) \partial^{l}\left(r^{-2 / 3}\right) \\
& +\frac{4}{9} H r^{-2} \partial_{l}(r) \partial^{l}(r) .
\end{aligned}
$$

Using that $H=r^{-2 / 3} f^{-1}$,

$$
\mathcal{G}_{\alpha \beta} X^{\beta} \partial_{l}\left(X^{\alpha}\right)=\frac{2}{3} r^{-1} \partial_{l} r
$$

and

we find that

$$
H \mathcal{G}_{00} \partial_{l}\left(X^{0}\right) \partial^{l}\left(X^{0}\right)=\frac{8}{9} H r^{-2} \partial_{l}(r) \partial^{l}(r)
$$

$$
H g_{\alpha \beta} \partial_{l}\left(j^{\alpha}\right) \partial^{l}\left(j^{\beta}\right)=r^{-2 / 3} f^{-1} \mathcal{G}_{\alpha \beta} \partial_{l}\left(X^{\alpha}\right) \partial^{l}\left(X^{\beta}\right)-\frac{1}{2} r^{-2 / 3} f^{-1} \mathcal{G}_{00} \partial_{l}\left(X^{0}\right) \partial^{l}\left(X^{0}\right) .
$$

Lastly, we reduce the second term of the right-hand side of the flow equation (5.13). Using (4.40) and (4.43) we can expand

$$
-\frac{1}{4} C_{I J K} X^{I} \Theta^{J} \cdot \Theta^{K}=-\frac{1}{2} \Omega_{\alpha \beta} r^{-4 / 3} \mathcal{G}^{+\alpha} \cdot \mathcal{G}^{+\beta}+r^{2 / 3} j_{\alpha} \tilde{d} \beta \cdot \mathcal{G}^{+\alpha} .
$$

For the first term at the right-hand side of (E.18) we use the reduced Einstein equation (4.47):

$$
\frac{1}{3} \Omega_{\alpha \beta} \mathcal{G}^{+\alpha} \cdot \mathcal{G}^{+\beta}=\tilde{\nabla}^{2}\left(f^{-1} X_{0}\right)=-\frac{2}{3} \tilde{\nabla}^{l}\left(f^{-1} \mathcal{G}_{00} E_{l}^{0}\right)
$$

where the second equality follows using (E.3) and the definition of $E_{l}^{0}$. Inserting $\mathcal{G}_{00}$ and using again the definition of $E_{l}^{0}$ we find that

$$
\begin{aligned}
-\frac{1}{2} \Omega_{\alpha \beta} r^{-4 / 3} \mathcal{G}^{+\alpha} \cdot \mathcal{G}^{+\beta}= & r^{-4 / 3} \tilde{\nabla}^{l}\left(f^{-1} \mathcal{G}_{00} E_{l}^{0}\right) \\
= & \frac{1}{2} \tilde{\nabla}^{l}\left(f^{-1} r^{4 / 3} E_{l}^{0}\right)+\frac{1}{2} f^{-1} \partial_{l}\left(X^{0}\right) \partial^{l}\left(r^{4 / 3}\right)+\frac{1}{2} f^{-2} X^{0} \partial_{l}(f) \partial^{l}\left(r^{4 / 3}\right) \\
= & \frac{1}{2} \tilde{\nabla}^{l}\left(f^{-1} r^{4 / 3} E_{l}^{0}\right)-f^{-1} \mathcal{G}_{00} \partial_{l}\left(X^{0}\right) \partial_{l}\left(X^{0}\right) \\
& +\frac{2}{3} r^{1 / 3} f^{-2} X^{0} \partial_{l}(f) \partial^{l}(r) .
\end{aligned}
$$

Substitution of (E.20) in (E.18) yields

$$
\begin{aligned}
-j_{\alpha} \tilde{d} \beta \cdot \mathcal{G}^{+\alpha}=-r^{-2 / 3}[ & -\frac{1}{4} C_{I J K} X^{I} \Theta^{J} \cdot \Theta^{K}-\frac{1}{2} \tilde{\nabla}^{l}\left(f^{-1} r^{4 / 3} E_{l}^{0}\right) \\
& \left.+f^{-1} \mathcal{G}_{00} \partial_{l}\left(X^{0}\right) \partial^{l}\left(X^{0}\right)-\frac{2}{3} f^{-2} r^{-1} \partial_{l}(f) \partial^{l}(r)\right] .
\end{aligned}
$$


Substitution of (E.13), (E.17) and (E.21) in (5.13) yields

$$
\tilde{\nabla}^{l}\left(f^{-1} \mathcal{G}_{I J} X^{I} E_{l}^{J}\right)=f^{-1} \mathcal{G}_{I J} \partial_{l}\left(X^{I}\right) \partial^{l}\left(X^{J}\right)-\frac{1}{4} C_{I J K} X^{I} \Theta^{J} \cdot \Theta^{K} .
$$

This is the five-dimensional flow equation.

Open Access. This article is distributed under the terms of the Creative Commons Attribution License (CC-BY 4.0), which permits any use, distribution and reproduction in any medium, provided the original author(s) and source are credited.

\section{References}

[1] R. Emparan and H.S. Reall, Black Holes in Higher Dimensions, Living Rev. Rel. 11 (2008) 6 [arXiv:0801.3471] [INSPIRE].

[2] H.K. Kunduri and J. Lucietti, Classification of near-horizon geometries of extremal black holes, Living Rev. Rel. 16 (2013) 8 [arXiv:1306.2517] [INSPIRE].

[3] R. Emparan and H.S. Reall, A rotating black ring solution in five-dimensions, Phys. Rev. Lett. 88 (2002) 101101 [hep-th/0110260] [INSPIRE].

[4] H.K. Kunduri and J. Lucietti, Supersymmetric Black Holes with Lens-Space Topology, Phys. Rev. Lett. 113 (2014) 211101 [arXiv:1408.6083] [INSPIRE].

[5] H.K. Kunduri and J. Lucietti, Black lenses in string theory, Phys. Rev. D 94 (2016) 064007 [arXiv: 1605.01545] [INSPIRE].

[6] S. Tomizawa and M. Nozawa, Supersymmetric black lenses in five dimensions, Phys. Rev. D 94 (2016) 044037 [arXiv: 1606.06643] [InSPIRE].

[7] J.B. Gutowski, D. Martelli and H.S. Reall, All supersymmetric solutions of minimal supergravity in six dimensions, Class. Quant. Grav. 20 (2003) 5049 [hep-th/0306235] [INSPIRE].

[8] M. Akyol and G. Papadopoulos, Topology and geometry of 6-dimensional $(1,0)$ supergravity black hole horizons, Class. Quant. Grav. 29 (2012) 055002 [arXiv:1109.4254] [INSPIRE].

[9] A. Strominger and C. Vafa, Microscopic origin of the Bekenstein-Hawking entropy, Phys. Lett. B 379 (1996) 99 [hep-th/9601029] [INSPIRE].

[10] J.C. Breckenridge, R.C. Myers, A.W. Peet and C. Vafa, D-branes and spinning black holes, Phys. Lett. B 391 (1997) 93 [hep-th/9602065] [INSPIRE].

[11] I. Bena, S. Giusto, M. Shigemori and N.P. Warner, Supersymmetric Solutions in Six Dimensions: A Linear Structure, JHEP 03 (2012) 084 [arXiv:1110.2781] [INSPIRE].

[12] B.E. Niehoff and N.P. Warner, Doubly-Fluctuating BPS Solutions in Six Dimensions, JHEP 10 (2013) 137 [arXiv: 1303.5449] [INSPIRE].

[13] S. Giusto, L. Martucci, M. Petrini and R. Russo, $6 D$ microstate geometries from 10D structures, Nucl. Phys. B 876 (2013) 509 [arXiv:1306.1745] [inSPIRE].

[14] I. Bena, S. Giusto, R. Russo, M. Shigemori and N.P. Warner, Habemus Superstratum! A constructive proof of the existence of superstrata, JHEP 05 (2015) 110 [arXiv:1503.01463] [INSPIRE].

[15] P. de Lange, D.R. Mayerson and B. Vercnocke, Structure of Six-Dimensional Microstate Geometries, JHEP 09 (2015) 075 [arXiv:1504.07987] [INSPIRE]. 
[16] I. Bena et al., Asymptotically-flat supergravity solutions deep inside the black-hole regime, JHEP 02 (2018) 014 [arXiv: 1711.10474] [INSPIRE].

[17] M. Cariglia and O.A.P. Mac Conamhna, The general form of supersymmetric solutions of $N=(1,0) \mathrm{U}(1)$ and $\mathrm{SU}(2)$ gauged supergravities in six-dimensions, Class. Quant. Grav. 21 (2004) 3171 [hep-th/0402055] [INSPIRE].

[18] D. Martelli and J.F. Morales, Bubbling AdS 3 , JHEP 02 (2005) 048 [hep-th/0412136] [INSPIRE].

[19] N. Bobev, B.E. Niehoff and N.P. Warner, New Supersymmetric Bubbles on $A d S_{3} \times S^{3}$, JHEP 10 (2012) 013 [arXiv: 1204.1972] [INSPIRE].

[20] B.E. Niehoff, O. Vasilakis and N.P. Warner, Multi-Superthreads and Supersheets, JHEP 04 (2013) 046 [arXiv:1203.1348] [INSPIRE].

[21] C. Vafa, Black holes and Calabi-Yau threefolds, Adv. Theor. Math. Phys. 2 (1998) 207 [hep-th/9711067] [INSPIRE].

[22] B. Haghighat, S. Murthy, C. Vafa and S. Vandoren, F-Theory, Spinning Black Holes and Multi-string Branches, JHEP 01 (2016) 009 [arXiv: 1509.00455] [INSPIRE].

[23] C. Lawrie, S. Schäfer-Nameki and T. Weigand, Chiral 2d theories from $N=4 S Y M$ with varying coupling, JHEP 04 (2017) 111 [arXiv:1612.05640] [INSPIRE].

[24] C. Couzens, C. Lawrie, D. Martelli, S. Schäfer-Nameki and J.-M. Wong, F-theory and $A d S_{3} / C F T_{2}, J H E P 08$ (2017) 043 [arXiv: 1705.04679] [INSPIRE].

[25] C. Couzens, D. Martelli and S. Schäfer-Nameki, F-theory and $A d S_{3} / C F T_{2}(2,0)$, arXiv: 1712.07631 [INSPIRE].

[26] C. Vafa, Evidence for F-theory, Nucl. Phys. B 469 (1996) 403 [hep-th/9602022] [inSPIRE].

[27] D.R. Morrison and C. Vafa, Compactifications of F-theory on Calabi-Yau threefolds. 1, Nucl. Phys. B 473 (1996) 74 [hep-th/9602114] [InSPIRE].

[28] D.R. Morrison and C. Vafa, Compactifications of F-theory on Calabi-Yau threefolds. 2, Nucl. Phys. B 476 (1996) 437 [hep-th/9603161] [INSPIRE].

[29] F. Bonetti and T.W. Grimm, Six-dimensional $(1,0)$ effective action of F-theory via M-theory on Calabi-Yau threefolds, JHEP 05 (2012) 019 [arXiv:1112.1082] [INSPIRE].

[30] F. Bonetti, T.W. Grimm and S. Hohenegger, One-loop Chern-Simons terms in five dimensions, JHEP 07 (2013) 043 [arXiv: 1302.2918] [INSPIRE].

[31] G.W. Gibbons and C.M. Hull, A Bogomolny Bound for General Relativity and Solitons in $N=2$ Supergravity, Phys. Lett. B 109 (1982) 190 [InSPIRE].

[32] K.p. Tod, All Metrics Admitting Supercovariantly Constant Spinors, Phys. Lett. B 121 (1983) 241 [INSPIRE].

[33] K.P. Tod, More on supercovariantly constant spinors, Class. Quant. Grav. 12 (1995) 1801 [INSPIRE].

[34] F. Denef, Supergravity flows and D-brane stability, JHEP 08 (2000) 050 [hep-th/0005049] [INSPIRE].

[35] P. Meessen and T. Ortín, The supersymmetric configurations of $N=2, D=4$ supergravity coupled to vector supermultiplets, Nucl. Phys. B 749 (2006) 291 [hep-th/0603099] [INSPIRE].

[36] M. Huebscher, P. Meessen and T. Ortín, Supersymmetric solutions of $N=2 D=4$ SUGRA: The whole ungauged shebang, Nucl. Phys. B 759 (2006) 228 [hep-th/0606281] [INSPIRE]. 
[37] J.P. Gauntlett, J.B. Gutowski, C.M. Hull, S. Pakis and H.S. Reall, All supersymmetric solutions of minimal supergravity in five dimensions, Class. Quant. Grav. 20 (2003) 4587 [hep-th/0209114] [INSPIRE].

[38] J.P. Gauntlett and J.B. Gutowski, All supersymmetric solutions of minimal gauged supergravity in five dimensions, Phys. Rev. D 68 (2003) 105009 [Erratum ibid. D 70 (2004) 089901] [hep-th/0304064] [INSPIRE].

[39] J.B. Gutowski and H.S. Reall, General supersymmetric AdS $S_{5}$ black holes, JHEP 04 (2004) 048 [hep-th/0401129] [INSPIRE].

[40] J. Bellorín, P. Meessen and T. Ortín, All the supersymmetric solutions of $N=1, d=5$ ungauged supergravity, JHEP 01 (2007) 020 [hep-th/0610196] [INSPIRE].

[41] M. Akyol and G. Papadopoulos, Spinorial geometry and Killing spinor equations of $6 D$ supergravity, Class. Quant. Grav. 28 (2011) 105001 [arXiv:1010.2632] [INSPIRE].

[42] G.W. Gibbons and S.W. Hawking, Gravitational Multi-Instantons, Phys. Lett. B 78 (1978) 430 [INSPIRE].

[43] P.M. Crichigno, F. Porri and S. Vandoren, Bound states of spinning black holes in five dimensions, JHEP 05 (2017) 101 [arXiv: 1603.09729] [INSPIRE].

[44] S. Ferrara, R. Kallosh and A. Strominger, $N=2$ extremal black holes, Phys. Rev. D 52 (1995) R5412 [hep-th/9508072] [INSPIRE].

[45] S. Ferrara and R. Kallosh, Supersymmetry and attractors, Phys. Rev. D 54 (1996) 1514 [hep-th/9602136] [INSPIRE].

[46] L. Andrianopoli, S. Ferrara, A. Marrani and M. Trigiante, Non-BPS Attractors in $5 d$ and $6 d$ Extended Supergravity, Nucl. Phys. B 795 (2008) 428 [arXiv:0709.3488] [INSPIRE].

[47] S. Ferrara, A. Marrani, J.F. Morales and H. Samtleben, Intersecting Attractors, Phys. Rev. D 79 (2009) 065031 [arXiv:0812.0050] [INSPIRE].

[48] A. de Antonio Martin, T. Ortín and C.S. Shahbazi, The FGK formalism for black p-branes in d dimensions, JHEP 05 (2012) 045 [arXiv: 1203.0260] [INSPIRE].

[49] P.A. Cano and T. Ortín, All the supersymmetric solutions of ungauged $\mathcal{N}=(1,0), d=6$ supergravity, arXiv: 1804.04945 [INSPIRE].

[50] L.J. Romans, Selfduality for Interacting Fields: Covariant Field Equations for Six-dimensional Chiral Supergravities, Nucl. Phys. B 276 (1986) 71 [InSPIRE].

[51] A. Sagnotti, A note on the Green-Schwarz mechanism in open string theories, Phys. Lett. B 294 (1992) 196 [hep-th/9210127] [INSPIRE].

[52] S. Ferrara, F. Riccioni and A. Sagnotti, Tensor and vector multiplets in six-dimensional supergravity, Nucl. Phys. B 519 (1998) 115 [hep-th/9711059] [InSPIRE].

[53] P. Pasti, D.P. Sorokin and M. Tonin, On Lorentz invariant actions for chiral $p$ forms, Phys. Rev. D 55 (1997) 6292 [hep-th/9611100] [INSPIRE].

[54] G. Dall'Agata, K. Lechner and M. Tonin, Covariant actions for $N=1, D=6$ supergravity theories with chiral bosons, Nucl. Phys. B 512 (1998) 179 [hep-th/9710127] [INSPIRE].

[55] J. Ford, S. Giusto and A. Saxena, A class of BPS time-dependent 3-charge microstates from spectral flow, Nucl. Phys. B 790 (2008) 258 [hep-th/0612227] [INSPIRE].

[56] O. Lunin, S.D. Mathur and D. Turton, Adding momentum to supersymmetric geometries, Nucl. Phys. B 868 (2013) 383 [arXiv:1208.1770] [INSPIRE]. 
[57] M. Shigemori, Perturbative 3-charge microstate geometries in six dimensions, JHEP 10 (2013) 169 [arXiv:1307.3115] [INSPIRE].

[58] M. Günaydin, G. Sierra and P.K. Townsend, The Geometry of $N=2$ Maxwell-Einstein Supergravity and Jordan Algebras, Nucl. Phys. B 242 (1984) 244 [InSPIRE].

[59] J.P. Gauntlett and J.B. Gutowski, General concentric black rings, Phys. Rev. D 71 (2005) 045002 [hep-th/0408122] [INSPIRE].

[60] G.W. Gibbons and P.J. Ruback, The Hidden Symmetries of Multicenter Metrics, Commun. Math. Phys. 115 (1988) 267 [INSPIRE].

[61] I. Bena and N.P. Warner, Bubbling supertubes and foaming black holes, Phys. Rev. D 74 (2006) 066001 [hep-th/0505166] [INSPIRE].

[62] I. Bena, N. Bobev and N.P. Warner, Spectral Flow and the Spectrum of Multi-Center Solutions, Phys. Rev. D 77 (2008) 125025 [arXiv:0803.1203] [INSPIRE].

[63] I. Bena and N.P. Warner, Black holes, black rings and their microstates, Lect. Notes Phys. 755 (2008) 1 [hep-th/0701216] [INSPIRE].

[64] I. Bena, D.-E. Diaconescu and B. Florea, Black string entropy and Fourier-Mukai transform, JHEP 04 (2007) 045 [hep-th/0610068] [INSPIRE].

[65] J.M. Maldacena, A. Strominger and E. Witten, Black hole entropy in M-theory, JHEP 12 (1997) 002 [hep-th/9711053] [INSPIRE].

[66] H. het Lam and S. Vandoren, work in progress.

[67] H. Elvang, R. Emparan, D. Mateos and H.S. Reall, A supersymmetric black ring, Phys. Rev. Lett. 93 (2004) 211302 [hep-th/0407065] [INSPIRE].

[68] H. Elvang, R. Emparan, D. Mateos and H.S. Reall, Supersymmetric black rings and three-charge supertubes, Phys. Rev. D 71 (2005) 024033 [hep-th/0408120] [INSPIRE].

[69] G.T. Horowitz and D. Marolf, Counting states of black strings with traveling waves, Phys. Rev. D 55 (1997) 835 [hep-th/9605224] [INSPIRE].

[70] G.T. Horowitz and D. Marolf, Counting states of black strings with traveling waves. 2, Phys. Rev. D 55 (1997) 846 [hep-th/9606113] [INSPIRE].

[71] N. Kaloper, R.C. Myers and H. Roussel, Wavy strings: Black or bright?, Phys. Rev. D 55 (1997) 7625 [hep-th/9612248] [INSPIRE].

[72] G.T. Horowitz and H.-s. Yang, Black strings and classical hair, Phys. Rev. D 55 (1997) 7618 [hep-th/9701077] [INSPIRE].

[73] S.F. Ross, Singularities in wavy strings, JHEP 08 (1998) 003 [hep-th/9710158] [INSPIRE].

[74] P. Kraus and F. Larsen, Attractors and black rings, Phys. Rev. D 72 (2005) 024010 [hep-th/0503219] [INSPIRE].

[75] S. Ferrara, R. Minasian and A. Sagnotti, Low-energy analysis of $M$ and $F$ theories on Calabi-Yau threefolds, Nucl. Phys. B 474 (1996) 323 [hep-th/9604097] [INSPIRE]. 